\title{
An investigation of stress inaccuracies and proposed solution in the material point method
}

\author{
José León González Acosta ${ }^{1}$ Philip J. Vardon ${ }^{1}\left[0\right.$. Guido Remmerswaal ${ }^{1} \cdot$ Michael A. Hicks ${ }^{1}$
}

Received: 10 May 2019 / Accepted: 4 October 2019 / Published online: 14 November 2019

(c) The Author(s) 2019

\begin{abstract}
Stress inaccuracies (oscillations) are one of the main problems in the material point method (MPM), especially when advanced constitutive models are used. The origins of such oscillations are a combination of poor force and stiffness integration, stress recovery inaccuracies, and cell crossing problems. These are caused mainly by the use of shape function gradients and the use of material points for integration in MPM. The most common techniques developed to reduce stress oscillations consider adapting the shape function gradients so that they are continuous at the nodes. These techniques improve MPM, but problems remain, particularly in two and three dimensional cases. In this paper, the stress inaccuracies are investigated in detail, with particular reference to an implicit time integration scheme. Three modifications to MPM are implemented, and together these are able to remove almost all of the observed oscillations.
\end{abstract}

Keywords Double mapping $\cdot$ Material point method $\cdot$ Shape functions $\cdot$ Stress oscillation

\begin{tabular}{|c|c|c|c|}
\hline \multicolumn{2}{|c|}{ Abbreviations } & A & Constant derived from the axisymmetric \\
\hline \multicolumn{2}{|r|}{ Convected domain particle interpolation } & & solution \\
\hline CMPM & Composite material point method & $\mathrm{A}_{1}$ & Constant derived from the axisymmetric bound- \\
\hline DDMP & Dual domain material point & & ary conditions \\
\hline DM & Double mapping & $\mathbf{b}$ & Body forces \\
\hline DM-C & Double mapping using CMPM & $\mathbf{B}$ & Strain-displacement matrix \\
\hline \multirow{3}{*}{$\begin{array}{l}\text { DM-G } \\
\text { DM-GC }\end{array}$} & Double mapping using GIMP shape functions & $\mathbf{B}^{\mathrm{ax}}$ & Strain-displacement matrix for axisymmetric \\
\hline & Double mapping using GIMP shape functions & & domain \\
\hline & and CMPM & $\mathbf{B}^{\mathrm{C}}$ & Strain-displacement matrix for CMPM patch \\
\hline FEM & Finite element method & $c_{\mathrm{p}}$ & Soil peak cohesion \\
\hline & Finite element & $\mathrm{c}_{\mathrm{r}}$ & Soil residual cohesion \\
\hline \multirow{2}{*}{$\begin{array}{l}\text { GIMP } \\
\text { GM }\end{array}$} & Generalized interpolation material point & $\mathrm{C}$ & Constant derived from the axisymmetric \\
\hline & Gauss mapping & & solution \\
\hline \multirow{2}{*}{$\begin{array}{l}\text { MP } \\
\text { MPM }\end{array}$} & Material point & $\mathrm{cmp}$ & Current number of material points in the \\
\hline & Material point method & & element \\
\hline \multirow{2}{*}{$\begin{array}{l}\text { SD } \\
\text { SF }\end{array}$} & Material point support domain & $\mathrm{d}$ & Distance between the element boundary and the \\
\hline & Shape function & & axisymmetric internal boundary \\
\hline \multirow{2}{*}{\multicolumn{2}{|c|}{ Latin symbols }} & $\mathbf{D}_{\mathrm{g}}$ & Elastic matrix at the Gauss point \\
\hline & Acceleration vects & $\mathbf{D}_{\mathrm{i}}$ & Elastic matrix at node $\mathrm{i}$ \\
\hline \multirow{2}{*}{$\begin{array}{l}a \\
a_{p} \\
\bar{a}\end{array}$} & Material point acceleration & $\mathbf{D}_{\mathrm{p}}$ & Elastic matrix at the sampling point \\
\hline & Vector of nodal accelerations & $\mathbf{D}_{\mathrm{p}}^{\mathrm{ax}}$ & Elastic matrix at the sampling point for axisym- \\
\hline & & & Young's modul \\
\hline$\triangle$ Philip & Vardon & elmp & Material points affecting an element \\
\hline P.J.Va & lon@tudelft.nl & $\mathbf{F}^{\mathrm{ext}}$ & Vector of external nodal forces \\
\hline Sect & f Geo-Engineering, Faculty of Civil Enginee & $\mathbf{F}^{\text {int }}$ & Vector of internal nodal forces \\
\hline and & ciences, Delft University of Technology, Del & $\mathrm{F}_{\text {mag }}^{\text {int }}$ & Internal nodal force magnitude \\
\hline
\end{tabular}




\begin{tabular}{|c|c|c|c|}
\hline $\begin{array}{l}\mathrm{F}_{\mathrm{VM}} \\
\mathrm{F}_{\mathrm{x}}^{\text {int }}\end{array}$ & $\begin{array}{l}\text { Von Mises yield function } \\
\text { Nodal internal force in the horizontal direction }\end{array}$ & $r_{i}$ & $\begin{array}{l}\text { Internal boundary of the axisymmetric } \\
\text { benchmark }\end{array}$ \\
\hline $\begin{array}{l}F_{y}^{i n t} \\
F_{\text {yield }}\end{array}$ & $\begin{array}{l}\text { Nodal internal force in the vertical direction } \\
\text { Yield function }\end{array}$ & $\mathrm{r}_{\mathrm{mp} 1}$ & $\begin{array}{l}\text { Radial position of a material point at the bound- } \\
\text { ary of the axisymmetric benchmark }\end{array}$ \\
\hline g & Gravity vector & $r_{p}$ & Radial position of a material point \\
\hline$\underline{\mathrm{H}}$ & Height of the vertical cut benchmark & $\mathrm{s}$ & Internal boundary of the axisymmetric \\
\hline$\overline{\mathbf{H}}$ & Matrix of shape functions, representing either & & benchmark \\
\hline $\mathrm{H}_{\mathrm{s}}$ & $\begin{array}{l}\mathbf{N} \text { or matrix of } \mathrm{S}_{\mathrm{ip}^{*}} \\
\text { Softening modulus }\end{array}$ & smp & $\begin{array}{l}\text { Number of material points with a support } \\
\text { domain inside an element }\end{array}$ \\
\hline $\mathrm{i}$ & Subscript representing nodal values & $\mathrm{S}_{\mathrm{ip}}$ & GIMP shape functions \\
\hline $\mathbf{J}$ & Jacobian matrix & $\mathrm{S}_{\mathrm{ip} *}$ & Local GIMP shape functions \\
\hline $\mathbf{J}^{\mathrm{mp}}$ & $\begin{array}{l}\text { Jacobian matrix computed using material point } \\
\text { shape function derivatives }\end{array}$ & $t+\Delta t$ & $\begin{array}{l}\text { Superscript denoting value at current time step } \\
\text { Superscript denoting value at next time step }\end{array}$ \\
\hline$|\mathbf{J}|$ & Jacobian matrix determinant & $\mathrm{u}$ & Virtual displacement \\
\hline $\mathrm{k}$ & Iteration number & $\overline{\mathbf{u}}$ & Vector of nodal displacements \\
\hline $\mathbf{K}$ & Stiffness matrix & \multirow[t]{2}{*}{$\overline{\mathbf{u}}^{\mathrm{ext}}$} & Vector of nodal displacements in the extended \\
\hline $\mathbf{K}_{\mathrm{el}}$ & Element stiffness matrix & & domain using CMPM \\
\hline $\mathrm{K}_{\text {mag }}$ & Stiffness matrix magnitude & $\mathbf{v}$ & Velocity vector \\
\hline $\mathrm{K}_{\mathrm{x}}$ & $\begin{array}{l}\text { Diagonal entry of the stiffness matrix corre- } \\
\text { sponding to the horizontal degree of freedom }\end{array}$ & $\begin{array}{l}\overline{\mathbf{v}} \\
\mathrm{V}\end{array}$ & $\begin{array}{l}\text { Vector of nodal velocities } \\
\text { Body volume }\end{array}$ \\
\hline $\mathrm{K}_{\mathrm{y}}$ & $\begin{array}{l}\text { Diagonal entry of the stiffness matrix corre- } \\
\text { sponding to the vertical degree of freedom }\end{array}$ & $\begin{array}{l}V_{p} \\
\mathbf{v}_{\mathrm{p}}\end{array}$ & $\begin{array}{l}\text { Material point volume } \\
\text { Material point velocity }\end{array}$ \\
\hline $\mathbf{K}_{\mathrm{v}}$ & Global stiffness matrix & $\mathrm{W}$ & Material point integration weight \\
\hline $\operatorname{lp}$ & $\begin{array}{l}\text { Half of the material point support domain } \\
\text { length }\end{array}$ & $\begin{array}{l}\mathrm{W}^{\mathrm{FE}} \\
\mathrm{W}^{*}\end{array}$ & $\begin{array}{l}\text { Weight associated with the Gauss point } \\
\text { Modified material point integration weight }\end{array}$ \\
\hline $\begin{array}{l}\mathrm{L} \\
\mathbf{M}\end{array}$ & $\begin{array}{l}\text { Length of the vertical cut benchmark } \\
\text { Mass matrix }\end{array}$ & $\overline{\mathrm{W}}$ & $\begin{array}{l}\text { Material point weight, representing either } \mathrm{W} \text { or } \\
\mathrm{W}^{*}\end{array}$ \\
\hline $\mathrm{m}_{\mathrm{p}}$ & Material point mass & $\mathbf{x}^{\mathrm{C}}$ & Nodal coordinates of the CMPM patch \\
\hline $\mathrm{M}_{\mathrm{x}}$ & $\begin{array}{l}\text { Diagonal entry of the mass matrix correspond- } \\
\text { ing to the horizontal degree of freedom }\end{array}$ & $\begin{array}{l}\mathbf{x}_{\mathrm{g}} \\
\mathbf{x}_{\mathrm{p}}\end{array}$ & $\begin{array}{l}\text { Gauss position } \\
\text { Material point position }\end{array}$ \\
\hline $\mathrm{M}_{\mathrm{y}}$ & $\begin{array}{l}\text { Diagonal entry of the mass matrix correspond- } \\
\text { ing to the vertical degree of freedom }\end{array}$ & \multicolumn{2}{|c|}{ Greek symbols } \\
\hline $\mathrm{N}$ & Shape function & $\alpha$ & Newmark time stepping parameter \\
\hline $\mathbf{N}$ & Matrix of shape functions & $\chi_{\mathrm{p}}$ & Characteristic function \\
\hline $\mathrm{N}_{\mathrm{i}}$ & Nodal shape function & $\delta$ & Newmark time stepping parameter \\
\hline ngauss & Number of Gauss points in the element & $\delta_{\mathrm{mp}}$ & $\begin{array}{l}\text { Incremental displacement } \\
\text { Material point domain }\end{array}$ \\
\hline $\begin{array}{l}\mathrm{nmp} \\
\mathrm{nn}\end{array}$ & $\begin{array}{l}\text { Number of material points inside an element } \\
\text { Number of nodes }\end{array}$ & $\Delta \mathrm{q}$ & Incremental deviatoric stress \\
\hline & Matrix of global CMPM shape functions & $\Delta \mathrm{p}_{\mathrm{s}}$ & $\begin{array}{l}\text { Incremental applied pressure on the boundary } \\
\text { of the axisymmetric benchmark }\end{array}$ \\
\hline $\begin{array}{l}\mathbf{N}_{\text {local }}^{\mathrm{C}} \\
\mathrm{N}^{\mathrm{i}}\end{array}$ & $\begin{array}{l}\text { Matrix of local CMPM shape functions } \\
\text { CMPM shape functions, where } i \text { is the }\end{array}$ & $\Delta \mathrm{r}$ & $\begin{array}{l}\text { Mesh radial dimension for the axisymmetric } \\
\text { domain }\end{array}$ \\
\hline $\mathbf{N}^{\mathrm{i}}$ & $\begin{array}{l}\text { C-continuity } \\
\text { Matrix of CMPM shape functions, where i is } \\
\text { the C-continuity }\end{array}$ & $\begin{array}{l}\Delta \sigma_{\mathrm{p}} \\
\Delta \sigma_{\mathrm{m}} \\
\Delta \mathrm{t}\end{array}$ & $\begin{array}{l}\text { Stress increment vector at the material point } \\
\text { Incremental mean stress } \\
\text { Time step }\end{array}$ \\
\hline omp & $\begin{array}{l}\text { Original number of material points in the } \\
\text { element }\end{array}$ & $\Delta \overline{\mathbf{u}}$ & Vector of incremental nodal displacements \\
\hline $\mathrm{p}_{\mathrm{s}}$ & $\begin{array}{l}\text { Applied pressure on the boundary of the } \\
\text { axisymmetric benchmark }\end{array}$ & $\begin{array}{l}\Delta \mathrm{y} \\
\Delta \mathrm{x} \\
\Gamma\end{array}$ & $\begin{array}{l}\text { Mesh vertical dimension } \\
\text { Mesh horizontal dimension } \\
\text { Body surface }\end{array}$ \\
\hline $\mathrm{r}$ & Distance between the cylinder axis and any & $\eta$ & Vertical position in local coordinates \\
\hline$r_{e}$ & Outer boundary of the axisymmetric benchmark & 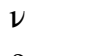 & Poisson's ratio \\
\hline & & $\rho_{\mathrm{p}}$ & Material point density \\
\hline
\end{tabular}




$\begin{array}{ll}\boldsymbol{\sigma} & \text { Cauchy stress tensor } \\ \sigma_{\mathrm{A}} & \text { Analytical radial stress } \\ \boldsymbol{\sigma}^{\mathrm{ax}} & \text { Cauchy stress tensor for axisymmetric domain } \\ \sigma_{\mathrm{L}} & \text { Stress inside a linear axisymmetric element } \\ \sigma_{\mathrm{Q}} & \text { Stress inside a quadratic axisymmetric element } \\ \sigma_{\theta} & \text { Tangential stress } \\ \sigma_{\mathrm{r}} & \text { Radial stress } \\ \sigma_{\mathrm{y}} & \text { Vertical stress } \\ \sigma_{\mathrm{xy}} & \text { Shear stress } \\ \boldsymbol{\tau} & \text { Traction at the surface } \\ \tau_{\mathrm{p}} & \text { Material point traction force } \\ \Omega & \text { Simulation domain } \\ \Omega_{\mathrm{p}} & \text { Material point support domain } \\ \xi & \text { Horizontal position in local coordinates }\end{array}$

\section{Introduction}

For many years, the finite element method (FEM) has been the most used numerical technique to analyse and design structures, but it is well known that it is often unable to handle large deformations. In FEM, the geometry of a problem is attached to a mesh, and if the mesh suffers large distortions, the analysis is unable to continue. The material point method (MPM) is a numerical technique that overcomes this limitation [28, 30], allowing for problems involving large deformations and multiple bodies to be analysed [5, 29, 33]. In MPM, the mechanical properties and the geometry of a problem are attached to a group of (material) points that move through an FEM mesh used to calculate the equation of motion in each time or load step. To enable this, the state variables are continuously mapped between the material points and the mesh. Many researchers have shown that MPM can be used to analyse some of the most common geotechnical problems, such as slope stability [1, 4, 9, 15, 31], foundation installation $[17,21,25]$ and soil anchors [12]. However, the accuracy of MPM, in particular relating to the stress fields, is still far from the desired level. Indeed, it is noted that many publications do not display full results of the stresses, either presenting only deformations or limited data, and that the majority of work presented in the literature so far uses simple constitutive models. In some work, the stress oscillations and inaccuracies are acknowledged, and mainly attributed to the use of discontinuous finite element (FE) shape function (SF) gradients (e.g. [3, 26, 27, 34]). Hence, a number of techniques have been developed to keep the SF gradients continuous between element boundaries, i.e. C1-continuous, for example:

- GIMP [6], which distributes the influence of each material point over a characteristic or support domain, possibly extending the influence to multiple elements at a time. Both the SF and the SF gradients are modified.
- CPDI [24], which is an extension of GIMP in which the material point support domain can deform, maintaining the interaction between particles even after large extension. There are a number of CPDI variants, with different orientations and behaviour of the support domain.

- B-spline MPM [26], which replaces the linear SFs by functions with higher-order B-spline basis functions, which are at least C1-continuous and positive definite.

- DDMP [34], which preserves the linear SFs and replaces the SF gradients by smooth continuous functions, thereby allowing the usage of a local integration procedure rather than having a material point support domain.

These techniques have been proven to reduce the impact of cell crossing. Meanwhile, other techniques use material point integration together with Gauss point integration to reduce numerical inaccuracies $[2,16]$. However, a complete investigation of the causes of the stress inaccuracies has not been presented. Moreover, these techniques typically involve explicit MPM schemes, thereby ignoring the errors the proposed solutions can cause in the integration of the stiffness matrix in implicit schemes and not exploiting the advantages of implicit time integration. Finally, examples have often been investigated only for 1D cases (usually with 2D elements), so that oscillations caused by other deformations, e.g. material rotation or distortion, have not been examined.

This paper first presents the theoretical background of implicit MPM. Then, two benchmark problems are introduced to illustrate the oscillation problem. In Sect. 4, the main causes of stress oscillations are investigated. Then, a series of existing and novel solutions are presented and investigated. Finally, a comparison of regular MPM and the new proposed oscillationfree MPM is given for the simulation of a vertical cut failure, in order to demonstrate the relative performance for a problem involving both $2 \mathrm{D}$ geometry and elasto-plasticity.

\section{Theoretical formulation}

MPM shares the same continuum mechanics background as FEM. The equation of conservation of momentum is given as

$\nabla \boldsymbol{\sigma}-\rho \mathbf{a}+\rho \mathbf{b}=0$

where $\boldsymbol{\sigma}$ is the symmetric Cauchy stress tensor, $\mathbf{a}$ is the acceleration, $\mathbf{b}$ are the body forces, and $\rho$ is the mass density. In MPM, because of the partition of unity of the SFs, mass is automatically conserved. The weak form of Eq. 1 including traction as a boundary condition is

$\int_{\mathrm{V}}(\boldsymbol{\sigma}(\nabla \mathrm{u})+\rho \mathbf{a u}-\rho \mathbf{b u}) \mathrm{dV}-\int_{\Gamma} \boldsymbol{\tau u d} \Gamma=0$ 
where $\mathrm{u}$ denotes the virtual displacement, $\boldsymbol{\tau}$ is the traction at the surface $\Gamma$ (i.e. the boundary condition), and $\mathrm{V}$ is the body volume. Following standard FEM discretisation, Eq. 2 can be expressed in the matrix form $[8,32]$

$\mathbf{M} \overline{\mathbf{a}}+\mathbf{K} \overline{\mathbf{u}}=\mathbf{F}^{\mathrm{ext}}-\mathbf{F}^{\mathrm{int}}$

where $\mathbf{M}$ is the mass matrix, $\mathbf{a}$ is the vector of nodal accelerations, $\mathbf{K}$ is the stiffness matrix, $\overline{\mathbf{u}}$ is the vector of nodal displacements, and $\mathbf{F}^{\text {ext }}$ and $\mathbf{F}^{\text {int }}$ are the external and internal force vectors, respectively. A quasi-static formulation is obtained by removing the Mā term from Eq. 3. Using the Gauss-Legendre quadrature rule and discretising the continuum body using a finite set of material points, the element (nodal) mass matrix can be expressed as

$\mathbf{M}=\sum_{\mathrm{p}=1}^{\mathrm{nmp}} \rho_{\mathrm{p}} \mathbf{N}\left(\mathbf{x}_{\mathrm{p}}\right)|\mathbf{J}| \mathrm{W}$

where $n m p$ is the number of material points in the element, $\rho_{\rho}$ is the material point density, $\mathbf{N}$ is the matrix of SFs evaluated at the material point position $\mathbf{x}_{\mathrm{p}}, \mathbf{J}$ is the Jacobian matrix, and $\mathrm{W}$ is the material point integration weight (which is dimensionless and equal to the volume of the material point in local coordinates).

The element stiffness matrix $\mathbf{K}$ can be expressed in terms of a small or large strain formulation, but for simplicity it is expressed here in the small strain formulation (for details of the large strain formulation, see [32]), as

$\mathbf{K}=\sum_{\mathrm{p}=1}^{\mathrm{nmp}} \mathbf{B}^{\mathrm{T}}\left(\mathbf{x}_{\mathrm{p}}\right) \mathbf{D}_{\mathrm{p}} \mathbf{B}\left(\mathbf{x}_{\mathrm{p}}\right)|\mathbf{J}| \mathrm{W}$

where $\mathbf{B}$ is the strain-displacement matrix and $\mathbf{D}_{\mathrm{p}}$ is the elastic matrix at the sampling point. The element (nodal) external forces $\mathbf{F}^{\text {ext }}$ considering gravity and boundary tractions are

$\mathbf{F}^{\mathrm{ext}}=\sum_{\mathrm{p}=1}^{\mathrm{nmp}} \rho_{\mathrm{p}} \mathbf{N}^{\mathrm{T}}\left(\mathbf{x}_{\mathrm{p}}\right) \mathbf{g}|\mathbf{J}| \mathrm{W}+\int_{\Gamma} \mathbf{N} \boldsymbol{\tau}_{\mathrm{p}} \mathrm{d} \Gamma$

where $\mathbf{g}$ is the gravity vector. The element (nodal) internal forces $\mathbf{F}^{\text {int }}$ are

$\mathbf{F}^{\text {int }}=\sum_{\mathrm{p}=1}^{\mathrm{nmp}} \boldsymbol{\sigma}_{\mathrm{p}} \mathbf{B}\left(\mathbf{x}_{\mathrm{p}}\right)|\mathbf{J}| \mathrm{W}$

where $\boldsymbol{\sigma}_{\mathrm{p}}$ is the vector of material point stresses. Details of the axisymmetric form of the previous equations are presented in "Appendix A1". Using Newmark's [20] time integration scheme,

$\overline{\mathbf{v}}^{\mathrm{t}+\Delta \mathrm{t}}=\overline{\mathbf{v}}^{\mathrm{t}}+\left[(1-\delta) \overline{\mathbf{a}}^{\mathrm{t}}+\delta \overline{\mathbf{a}}^{\mathrm{t}+\Delta \mathrm{t}}\right] \Delta \mathrm{t}$
$\overline{\mathbf{u}}^{\mathrm{t}+\Delta \mathrm{t}}=\overline{\mathbf{u}}^{\mathrm{t}}+\overline{\mathbf{v}}^{\mathrm{t}} \Delta \mathrm{t}+\left[\left(\frac{1}{2}-\alpha\right) \overline{\mathbf{a}}^{\mathrm{t}}+\alpha \overline{\mathbf{a}}^{\mathrm{t}+\Delta \mathrm{t}}\right] \Delta \mathrm{t}^{2}$

where $\Delta \mathrm{t}$ is the time step, $\overline{\mathbf{u}}^{\mathrm{t}+\Delta \mathrm{t}}, \overline{\mathbf{v}}^{\mathrm{t}+\Delta \mathrm{t}}$ and $\overline{\mathbf{a}}^{\mathrm{t}+\Delta \mathrm{t}}$ are the respective vectors of displacements, velocities and accelerations at time $\mathrm{t}+\Delta \mathrm{t}$, and $\alpha$ and $\delta$ are time stepping parameters that are chosen to be $\alpha=0.25$ and $\delta=0.5$ to give a constant-average-acceleration. Substituting Eq. 9 into Eq. 3 and rearranging leads to

$\left(\mathbf{K}^{\mathrm{t}}+\frac{\mathbf{M}^{\mathrm{t}}}{\alpha \Delta \mathrm{t}^{2}}\right) \Delta \overline{\mathbf{u}}=\mathbf{F}_{\mathrm{ext}}^{\mathrm{t}}+\mathbf{M}^{\mathrm{t}}\left(\frac{1}{\alpha \Delta \mathrm{t}} \overline{\mathbf{v}}^{\mathrm{t}}+\left(\frac{1}{2 \alpha}-1\right) \overline{\mathbf{a}}^{\mathrm{t}}\right)-\mathbf{F}_{\text {int }}^{\mathrm{t}}$

where $\Delta \overline{\mathbf{u}}=\overline{\mathbf{u}}^{\mathrm{t}+\Delta \mathrm{t}}-\overline{\mathbf{u}}^{\mathrm{t}}$ is the vector of incremental displacements. In "Appendix B", a study of the conservation of mass and momentum of the implicit MPM is analysed.

The above equation governs the behaviour of the body and it is therefore important to accurately evaluate each of the terms in order to ensure realistic behaviour. Following the solution of the updated displacements, the trial incremental stresses at the material points can be computed using the strain-displacement matrix as

$\Delta \boldsymbol{\sigma}_{\mathrm{p}}=\mathbf{D}_{\mathrm{p}} \mathbf{B}\left(\mathbf{x}_{\mathrm{p}}\right) \Delta \overline{\mathbf{u}}$

For an elasto-plastic material, stresses which are found to exceed the yield surface are redistributed using a consistent plastic return algorithm such that a new body force is calculated, and Eq. 10 is again solved to give plastic deformations. This is iteratively performed until no stresses exceed the yield surface. For more details see, for example, Bathe [8].

\subsection{Material point method}

MPM discretises the material into a series of (material) points which carry the information of the material (density, mass, deformation, velocity, acceleration and stresses). A mapping phase occurs at the start and at the end of each time or load step. At the beginning of each step the values required at the nodes in Eq. 10 (velocity, acceleration, etc.) are mapped via the SFs (see Wang et al. [32] for details). The matrices required for the calculation are calculated via element integration, as shown in Sect. 2, typically using material points as the sampling points. Afterwards, element assembly results in a set of global matrices representing nodal equations. A finite element calculation is then performed, with the state variables calculated at the nodes, in order to compute the deformation of the domain. Finally, another mapping step is undertaken to update the position and state variables of the material points. In Fig. 1, a sketch of the steps followed in MPM is shown. The SFs used to carry out the mapping and the integration are usually first 
Fig. 1 Steps followed in MPM. a Integration of material point variables to nodes at time $t$, b deformation of the domain as a result of the solution of the finite element calculation, and $\mathbf{c}$ update of material point variables and reset of the background mesh $(\mathrm{t}=\mathrm{t}+\Delta \mathrm{t})$ (a)

(b)

(c)

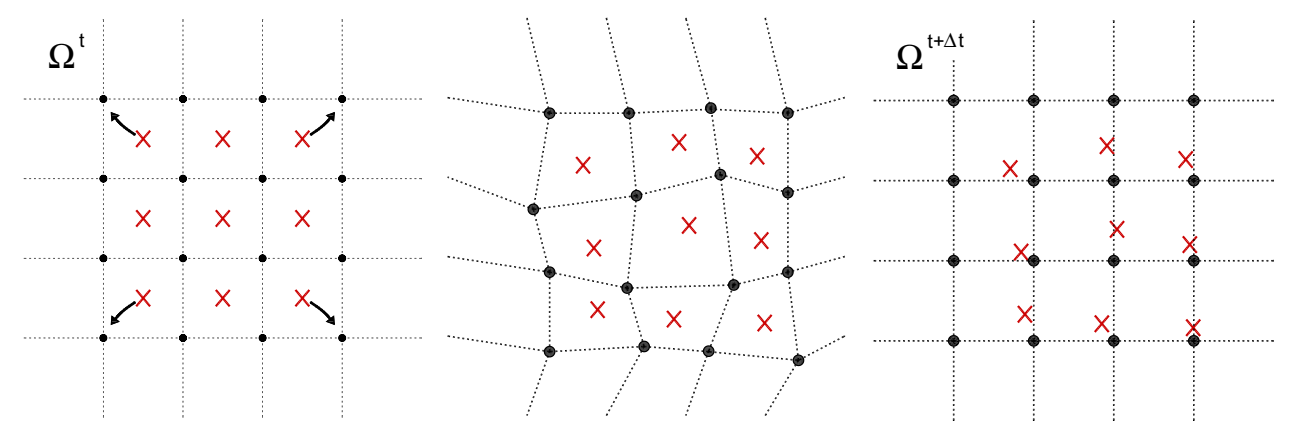

order (e.g. bi-linear in 2D) to avoid negative values which cause instability.

\section{Benchmarks}

Two benchmarks are introduced to demonstrate and investigate the inaccuracies which occur in MPM. The first benchmark consists of an elastic quasi-static axisymmetric problem. The second benchmark is a 2D dynamic, elastoplastic, vertical cut problem.

\subsection{Axisymmetric benchmark}

The first benchmark is similar to that presented by Naylor [19] and Mar and Hicks [18] to explore stress recovery. It consists of a hollow cylinder which deforms due to an incremental pressure $\left(\Delta \mathrm{p}_{\mathrm{s}}\right)$ applied on the internal boundary (s). The main benefit of this benchmark is that, unlike a 1D plane strain problem, the stresses inside the elements are not constant; moreover, they deviate from the real solution and, depending on the material point position, the deviation may be large or small.

Figure $2 \mathrm{a}, \mathrm{b}$ shows the initial conditions of the benchmark; that is, the top view of the cylinder and the finite element discretization of the cylinder wall, respectively. In both figures, the position of the boundary material point is shown (i.e. the material point nearest to the cylinder axis), which is used to determine the position of the boundary (s). Figure $2 \mathrm{c}$ and $d$ illustrate that, during the loading, the distance $r_{i}$ to the inner wall (s) changes, and is equal to the distance between the cylinder axis and the nearest active node (this implies that $r_{i}$ remains constant until the boundary material point jumps to the next element). To enable the numerical (large strain) solution to be interpreted in terms of the analytical (small strain) solution, the methodology includes the following three features: (1) the applied pressure $\Delta \mathrm{p}_{\mathrm{s}}$ on the
Fig. 2 Axisymmetric model of a hollow cylinder under internal pressure. a top view of the benchmark, b domain and boundary conditions, $\mathbf{c}$ initial internal boundary location, and d internal boundary location at a given step (a)

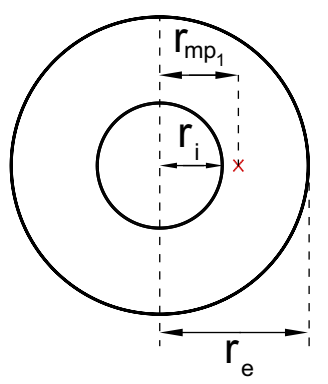

(c)

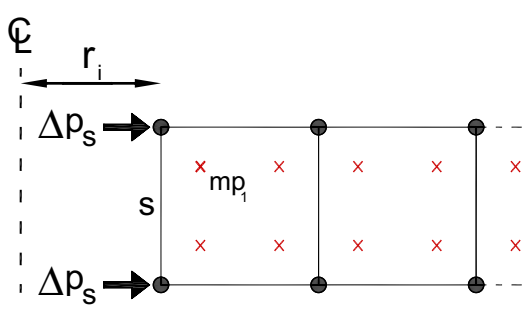

(b)

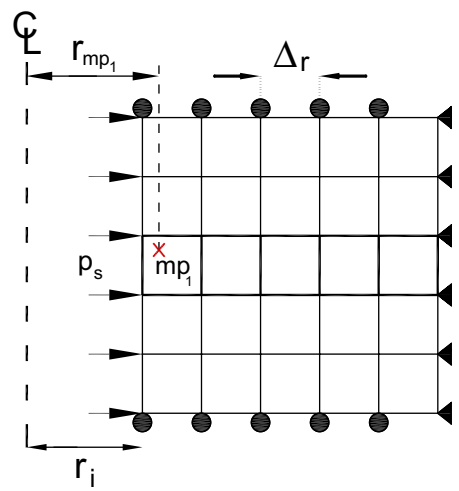

(d)

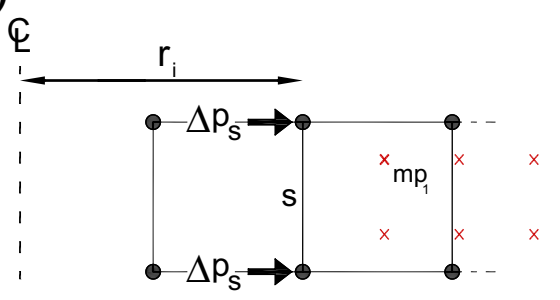




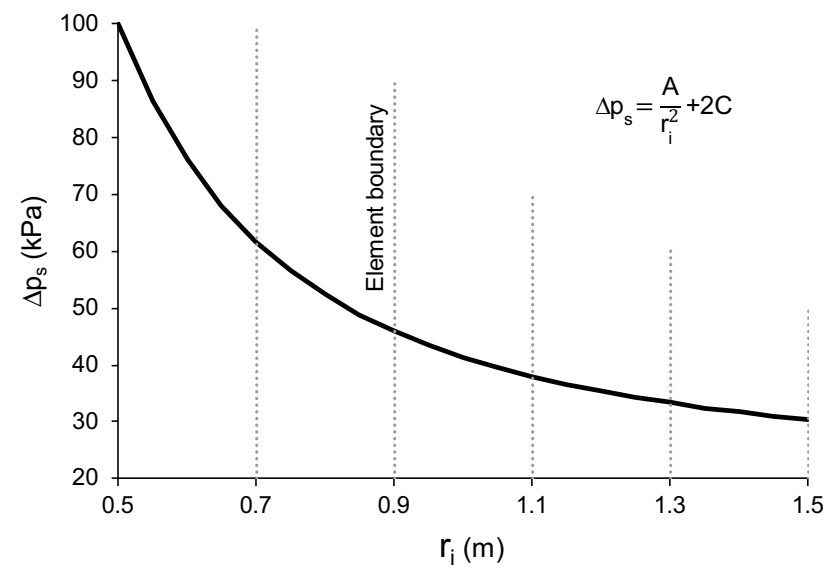

Fig. 3 Incremental pressure $\left(\Delta p_{s}\right)$ as a function of $r_{i}$

boundary (s) is applied to the outer nodes of the elements containing the outer most material points; (2) due to the new location of the inner wall, $\Delta \mathrm{p}_{\mathrm{s}}$ is re-evaluated as $\Delta \mathrm{p}_{\mathrm{s}}\left(\mathrm{r}_{\mathrm{i}}\right)=\mathrm{A} /$ $\mathrm{r}_{\mathrm{i}}^{2}+2 \mathrm{C}$, where $\mathrm{A}$ and $\mathrm{C}$ are constants associated with the initial geometry and boundary conditions of the benchmark, as shown in Fig. 3 (a description of the analytical solution and the constants $\mathrm{A}$ and $\mathrm{C}$ are presented in "Appendix A2"); (3) instead of accumulated stresses, incremental stresses at the material points are used throughout the analysis. These three features ensure that the incremental stress at the material points, for an arbitrary position of the cylinder wall, can be compared to the analytical stress related to the original geometry of the cylinder.

The inner (initial) and outer cylinder boundaries are located at $\mathrm{r}_{\mathrm{i}}=0.5 \mathrm{~m}$ and $\mathrm{r}_{\mathrm{e}}=1.5 \mathrm{~m}$, respectively. The cylinder domain is discretised by elements of dimension $\Delta \mathrm{r}=\Delta \mathrm{y}=0.20 \mathrm{~m}$, and each element initially contains four material points equally spaced. The elastic properties are Young's modulus, E $=1000 \mathrm{kPa}$, and Poisson's ratio, $\nu=0.30$. The initial applied pressure increment is $\Delta \mathrm{p}_{\mathrm{s}}=100 \mathrm{kPa}$, and $\mathrm{A}$ and $\mathrm{C}$ are $19.56 \mathrm{kN}$ and $10.87 \mathrm{kPa}$, respectively.

In Fig. 4, the incremental stress invariants (deviatoric stress $\Delta q$ and mean stress $\Delta \sigma_{m}$ ) at material point $m_{1}$ are plotted and compared to the analytical solution over $25 \Delta \mathrm{p}_{\mathrm{s}}$ increments. It is evident that the stress invariants can deviate strongly from the analytical solution.

\subsection{Vertical cut benchmark}

A 2D elasto-plastic vertical cut problem has been simulated using the Von Mises constitutive model incorporating postpeak softening as described in Wang et al. [32]. Figure 5 shows the domain, boundary conditions and discretisation. The height $\mathrm{H}$ of the cut and length $\mathrm{L}$ of the domain are $3.0 \mathrm{~m}$ and $6.0 \mathrm{~m}$, respectively; the element size is $\Delta x=\Delta y=0.10 \mathrm{~m}$ (a)

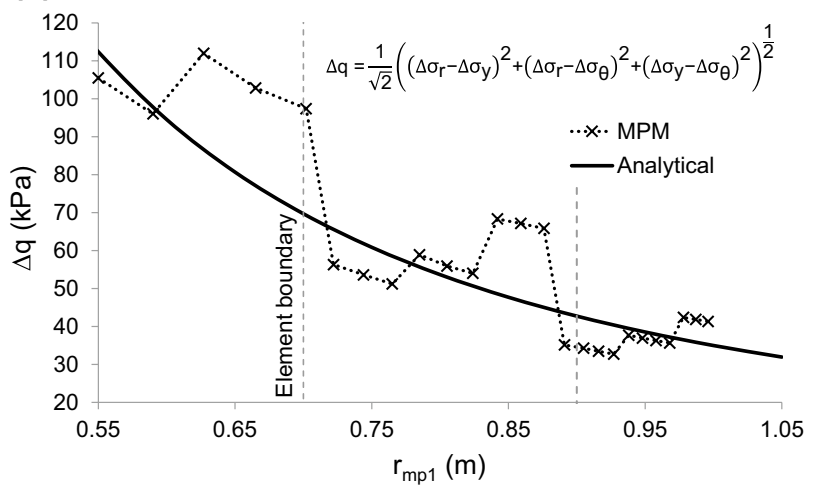

(b) 0

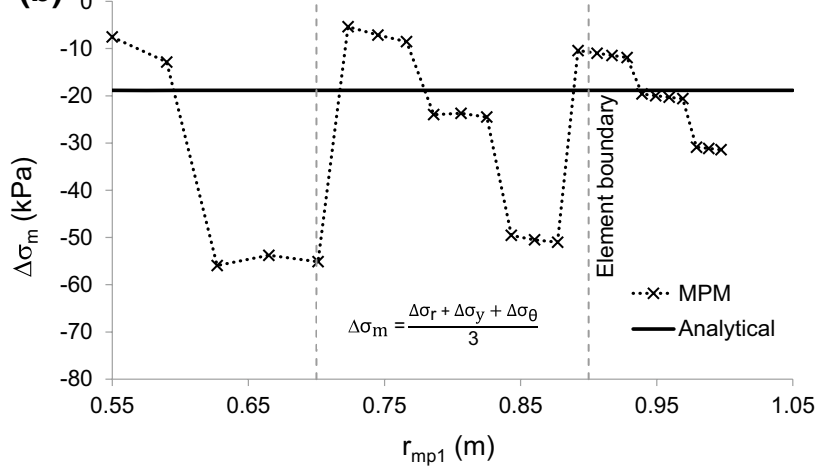

Fig. 4 Evolution of $\mathrm{mp}_{1}$ stresses relative to $r_{\mathrm{mp} 1}$. a deviatoric stress $\Delta \mathrm{q}$, and $\mathbf{b}$ mean stress $\Delta \sigma_{\mathrm{m}}$

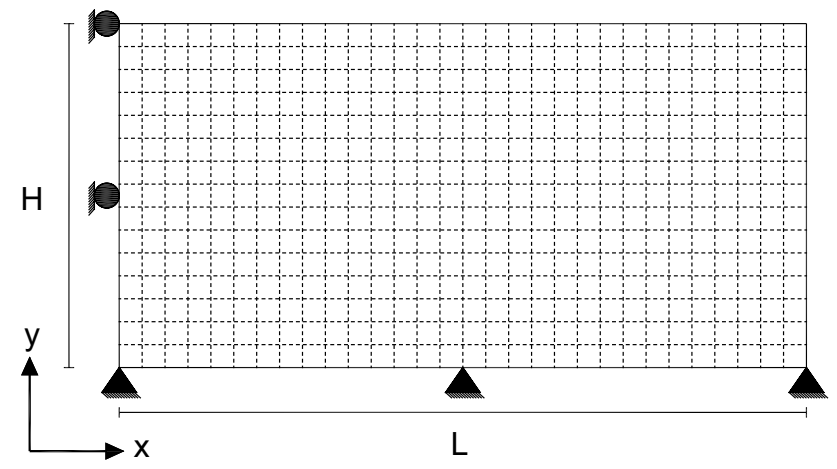

Fig. 5 Sketch of the cutting stability problem

and each element contains initially four equally distributed material points. The elastic parameters are $\mathrm{E}=1000 \mathrm{kPa}$ and $\nu=0.35$, whereas the peak cohesion is $c_{p}=12 \mathrm{kPa}$, the residual cohesion is $\mathrm{c}_{\mathrm{r}}=3 \mathrm{kPa}$, and the softening modulus is $H_{s}=-30 \mathrm{kPa}$. At the left boundary, the nodes are partly fixed to avoid displacement in the horizontal direction, whereas the nodes are fully fixed at the bottom boundary. The initial stresses in the domain are generated by fixing the locations of the material points and applying gravity 
loads until the internal and external forces are in equilibrium. After equilibrium is reached, the material points are released and deformation takes place.

Figure 6a, b shows contours of the deviatoric and mean stresses, respectively. It is seen that during the movement of material points, both deviatoric and mean stress oscillations occur, although the overall failure mechanism is as expected. For Fig. 6b, the shown range was fixed between 10 and $-30 \mathrm{kPa}$ because the oscillations are enormous in and around the shear band.

\section{Oscillations in MPM}

The MPM technique can be seen as an FE stepwise procedure, in which the integration points (now called material points) move together with the mesh, but keep their new positions while the mesh returns to its original position. This allows the simulation of large deformations since extreme distortion of the mesh is avoided, although the process is found to cause stress oscillations. There are a number of contributing factors causing these oscillations, which are investigated below.

\subsection{Stress recovery}

As is typical in many implicit FEM formulations, displacements have been used as the primary variable and stresses are back-calculated using the strain-displacement matrix and the elastic matrix (Eq. 11). During the back calculation of stresses, an oscillation occurs because the stresses inside the elements, interpolated using the element SF gradients, do not agree with the analytical stresses except at the superconvergent positions [7, 19, 35]. This problem is not observed in problems where the analytical stress is uniform across the element, e.g. as in a 1D bar. Figure 7 illustrates the radial stress inside a linear or quadratic axisymmetric element. It is seen that the computed stress distribution across the linear element $\left(\sigma_{\mathrm{L}}\right)$ is different from that across the quadratic element $\left(\sigma_{\mathrm{Q}}\right)$, and that both are different from the analytical stress $\left(\sigma_{\mathrm{A}}\right)$. However, the linear and quadratic stresses $\left(\sigma_{\mathrm{L}}\right.$ and $\sigma_{\mathrm{Q}}$, respectively) match the analytical solution exactly at the Gauss point locations. This means that, depending on the position of the material point, the recovered stresses can be either higher or lower than the analytical stresses, as illustrated in Fig. 4.

Figure 8 shows the analytical radial stress distribution and the stress recovered using MPM (or FEM) at any stress recovery position for the first load step in the axisymmetric benchmark. It is evident that the exact solution is near the centre of the elements, and recovering stresses at any
Fig. 6 MPM stresses after $1.0 \mathrm{~m}$ of horizontal displacement at the toe. a deviatoric stress, and b mean stress (a)
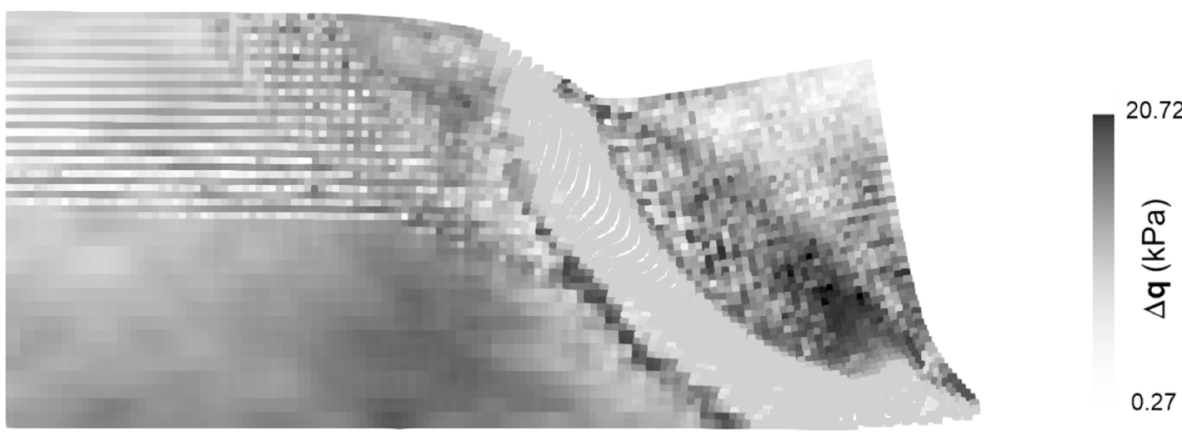

(b)

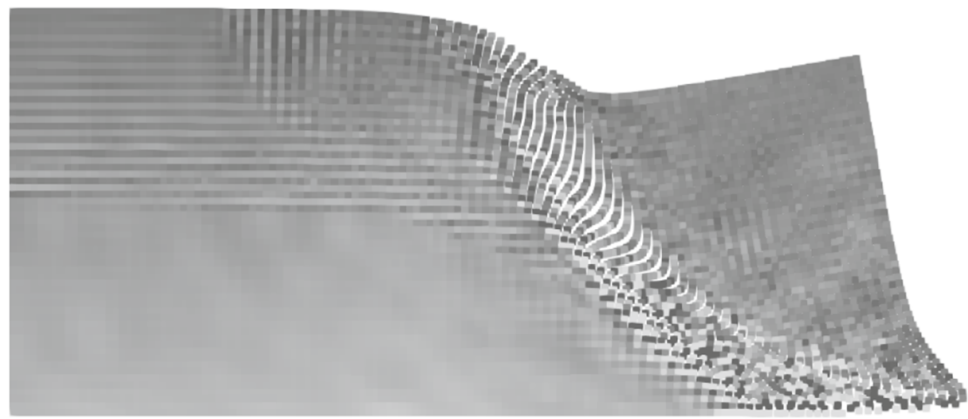


Fig. 7 Radial stress inside an axisymmetric element

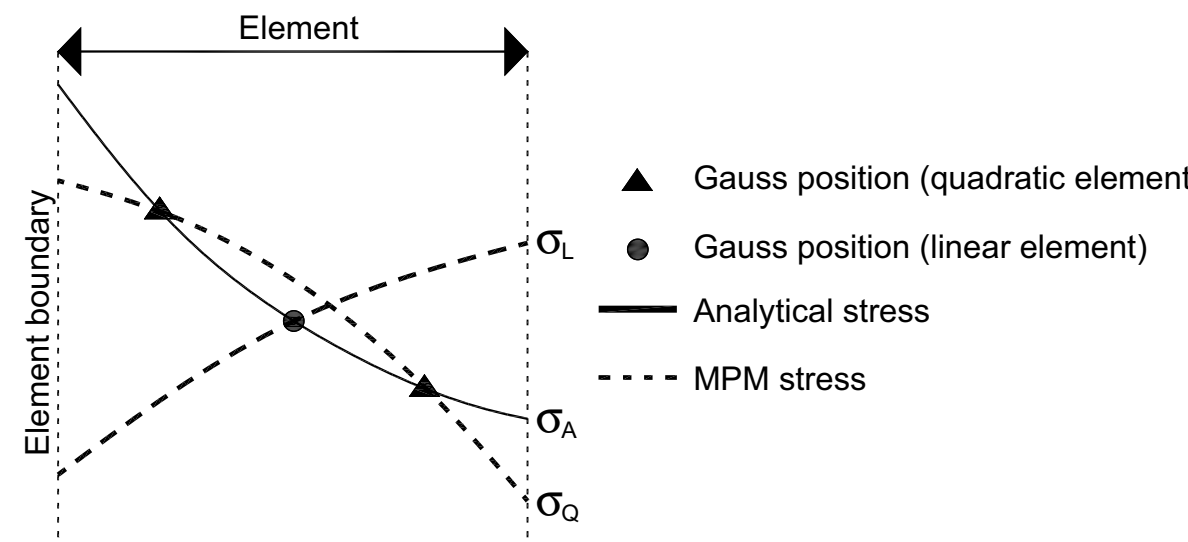

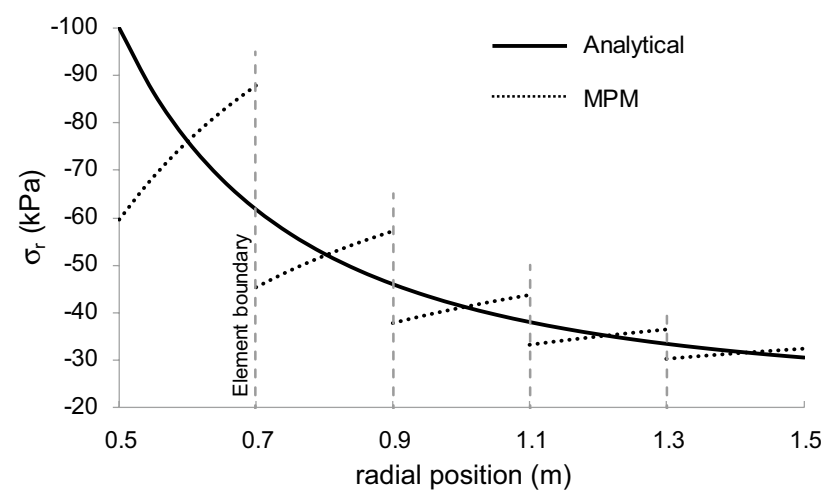

Fig. 8 Analytical radial stress and stresses recovered using MPM in the axisymmetric benchmark other position will cause oscillations. It can also be seen that there will be a large oscillation whenever a material point crosses an element boundary, since the radial stress is discontinuous across inter-element boundaries.

\subsection{Nodal integration using SF gradients}

The nodal integrations of $\mathbf{F}^{\text {int }}$ and $\mathbf{K}$ are performed using SF gradients and the material point positions. However, considering that the SF gradients used in MPM are bi-linear (linear elements) and discontinuous, and that the material point positions change each time step, the resulting nodal values are inaccurate, especially if material points cross element boundaries. Next, a description of the SF gradients in MPM and the consequences of using them are presented.
Fig. 9 a Element local numbering, b regular SF associated with node $1, \mathbf{c}$ horizontal SF gradient associated with node 1 , and $\mathbf{d}$ vertical SF gradient associated with node $1 . \mathrm{N}_{\mathrm{i}}$ is the shape function for node $i$, and $\xi$ and $\eta$ are local coordinates (a)

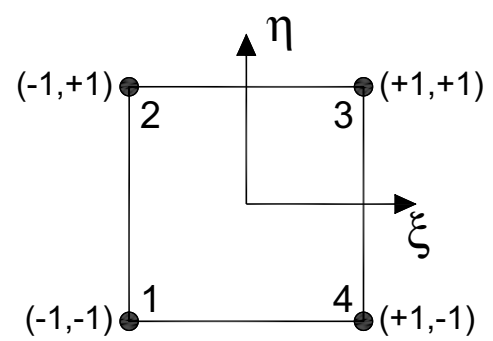

(c)

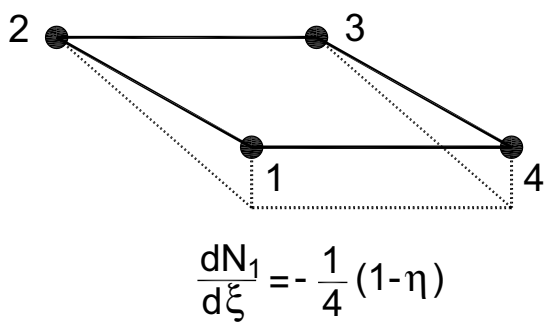

(b)

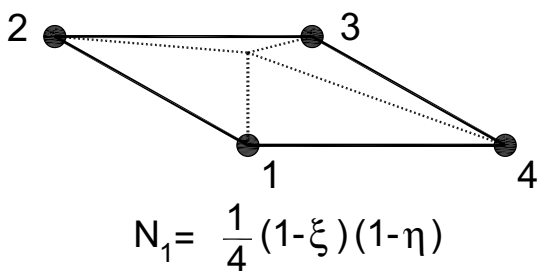

(d)

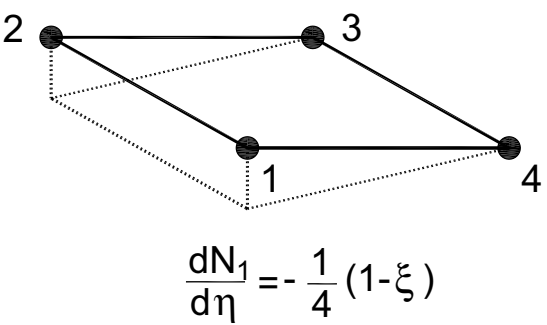




\subsubsection{D bi-linear shape functions}

Figure 9 shows the SF (Fig. 9b) and the horizontal and vertical SF gradients (Fig. 9c, d) of node 1 of a 4-node square element (Fig. 9a). It is noticed that the SF gradient is a maximum at the node, constant in the direction associated with the SF gradient, and decreases down to zero in the orthogonal direction. When a material point crosses an element boundary, the combination of the two element SFs must be considered.

In Fig. 10, two elements are shown: $E_{1}$ and $E_{2}$ (Fig. 10a). The SFs and SF gradients in both directions of node 5 are shown in Fig. 10b-d, respectively. Figure 10b shows that the SFs are continuous between elements, while Fig. 10d shows that the vertical SF gradient is continuous between elements in the horizontal direction and constant in the vertical direction. On the other hand, Fig. 10c shows that the horizontal SF gradients at the inter-element boundary are discontinuous, and that they decrease in the vertical direction.

(a)

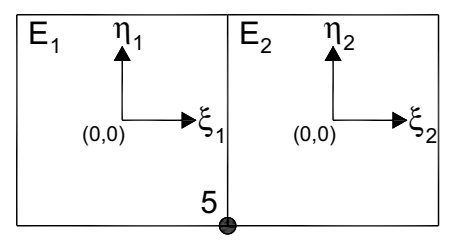

(b)

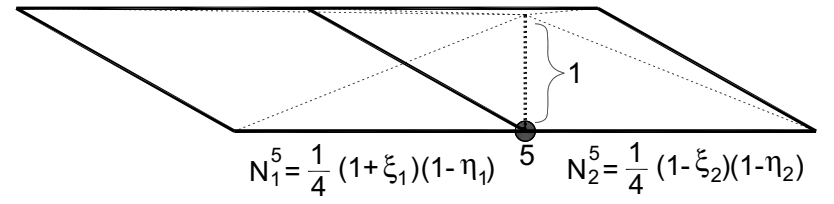

(c)

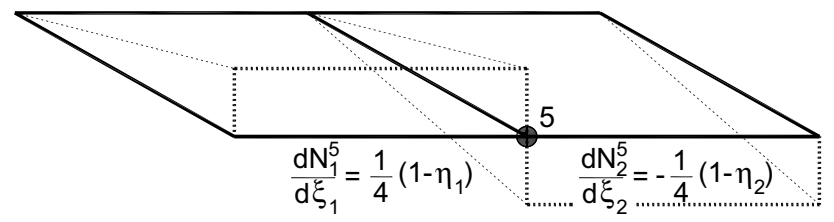

(d)

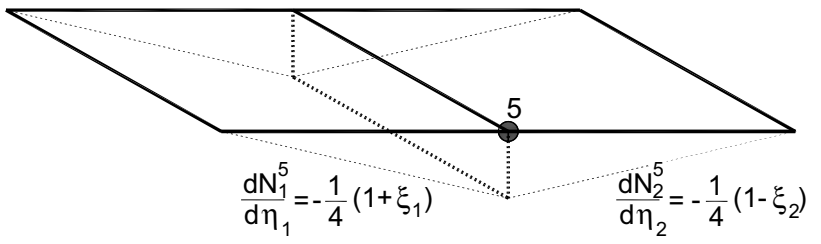

Fig. 10 a Connected elements $E_{1}$ and $E_{2}$, b regular SFs for node 5, c SF gradients in the horizontal direction, and $\mathbf{d}$ SF gradients in the vertical direction. In this figure, the superscript and subscript refer to the node and element numbering, respectively

\subsubsection{Integration of the internal forces $F^{\text {int }}$ and stiffness $K$}

Using SF gradients in the integration of any variable (i.e. $\mathbf{F}^{\text {int }}$ and $\mathbf{K}$ ) results in an inadequate nodal distribution, whereas, if regular SFs are used, the nodal distribution is smoother ( $\mathbf{M}$ and $\mathbf{F}^{\mathrm{ext}}$ ). Moreover, two differences should be noticed between the integration of $\mathbf{F}^{\text {int }}$ and $\mathbf{K}$. The first is that, to integrate $\mathbf{F}^{\text {int }}$, the strain-displacement matrix $(\mathbf{B})$ is used once (Eq. 7), whereas the element stiffness is computed using both $\mathbf{B}$ and its transpose $\mathbf{B}^{\mathrm{T}}$ (Eq. 5). The second is that to integrate $\mathbf{F}^{\text {int }}$, the stresses of the material points are used, whereas to integrate $\mathbf{K}$ the elastic properties of the material points are used. The significance of this is that the elastic properties are constant throughout the analysis, whereas the material point stresses change during the analysis, causing possible accumulation of errors.

As an example of the inaccuracies caused by using SF gradients, the vertical and horizontal nodal internal force distributions $\left(\mathrm{F}_{\mathrm{x}}^{\mathrm{int}}\right.$ and $\left.\mathrm{F}_{\mathrm{y}}^{\mathrm{int}}\right)$ and the diagonal entries of the stiffness matrix (Eq. 5) corresponding to the vertical and horizontal degrees of freedom $\left(\mathrm{K}_{\mathrm{x}}\right.$ and $\left.\mathrm{K}_{\mathrm{y}}\right)$ using two different material point distributions, are computed for nodes 1-5 of the plane strain finite element mesh shown in Fig. 11. In both cases the material points are equally distributed inside the elements; in the first case (Fig. 11a) the material points are located inside each element, whereas in the second case (Fig. 11b) the material points have moved and some are located at the inter-element boundaries. After the movement, the material points are still located inside their original element, except for material points a-d which have crossed the boundary by an infinitesimal distance. Stress components of $\sigma_{\mathrm{x}}=\sigma_{\mathrm{y}}=-1.0 \mathrm{MPa}$ and $\sigma_{\mathrm{xy}}=0$, a Young's modulus of $\mathrm{E}=1.0 \mathrm{kPa}$ and a Poisson's ratio of $\nu=0$ for each material point have been considered, while the distance between the nodes is $1 \mathrm{~m}$ and the material point weights are equal to 1 .

In Fig. 11c, d, the vertical internal force is equal to zero in both cases. The force is unchanged because the horizontal displacement of the material points does not affect the values of the vertical SF gradients, and equals zero because the internal vertical forces on both sides of the nodes are the same but with an opposite sign. However, the distribution of the horizontal internal force is highly inaccurate due to the material point crossing the element boundary and the discontinuity of the horizontal SF gradients (Fig. 11d). When integrating the nodal stiffness, the horizontal and vertical stiffnesses are initially similar (Fig. 11e). However, as the material points cross an element boundary (Fig. 11f), the inaccuracies are evident again, although they are smaller than those of the internal forces. This is because the product $\mathbf{B B}^{\mathrm{T}}$ returns positive nodal values, so avoiding the change in sign of the SF gradients. 
(a)

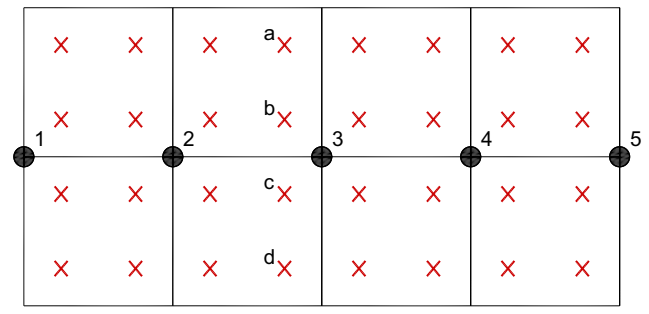

(c)

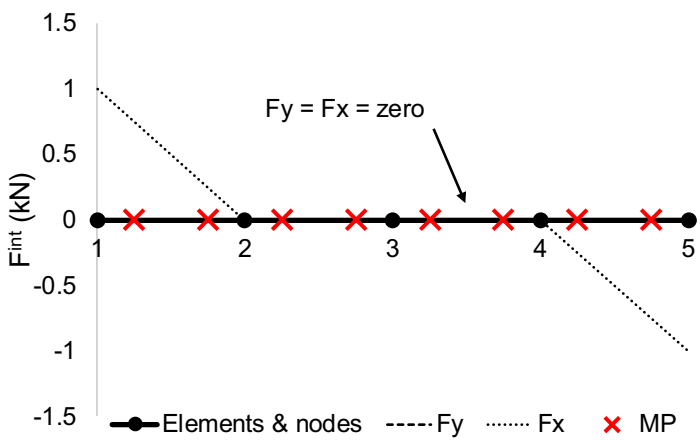

(e)

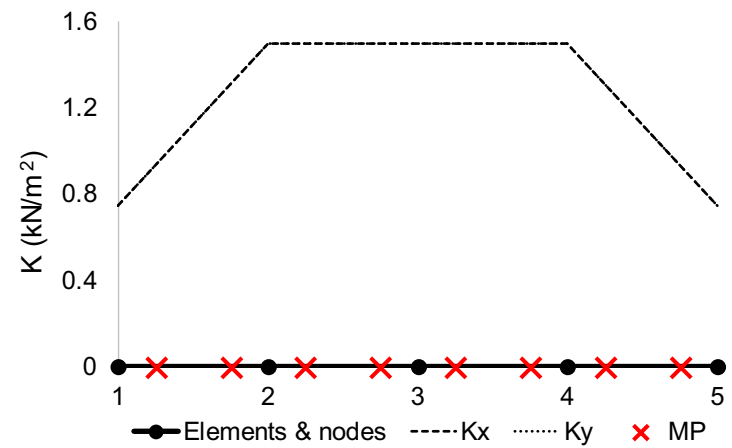

Fig. 11 Investigation of internal forces and stiffness calculation using a material points inside elements, and $\mathbf{b}$ displaced material points where some material points (e.g. a-d) have crossed the inter-element

\subsection{Nodal integration of the mass $M$ and external forces $F^{\text {ext }}$ using SFs}

The integration of $\mathbf{M}$ and $\mathbf{F}^{\mathrm{ext}}$ is performed using SFs rather than SF gradients, so that discontinuities between elements do not occur. In this example, only the external forces caused by gravity are considered. Since a lumped form of the mass matrix is used, and also because of the partition of unity of SFs, any initial distribution of material points inside the elements results in the same nodal mass (or external force), as long as the distribution is symmetrical. As an example, Fig. 12 shows two different material (b)

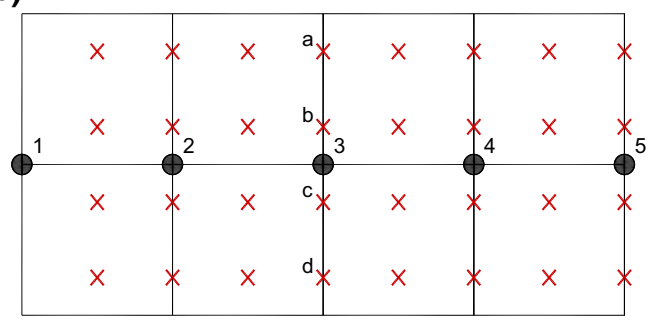

(d)

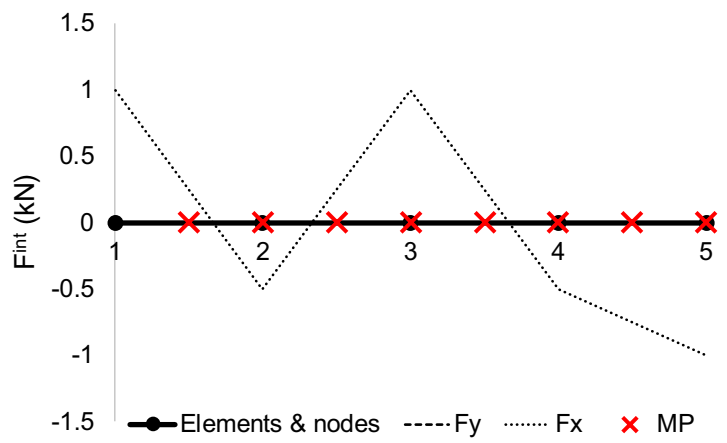

(f)

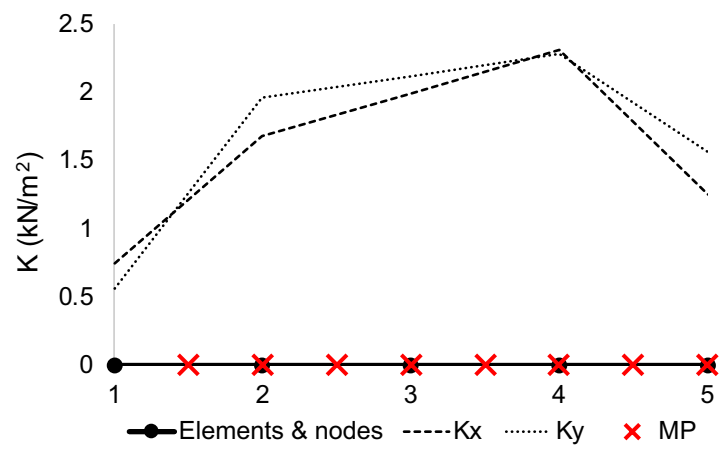

boundaries. Nodal force distribution $\mathbf{c}$ before boundary crossing and d after boundary crossing, and stiffness distribution e before boundary crossing and $\mathbf{f}$ after boundary crossing
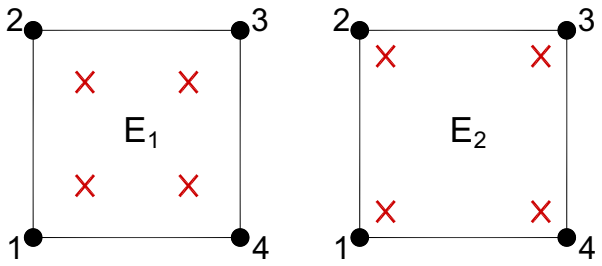

Fig. 12 Different symmetric material point distributions in two elements 
(a)

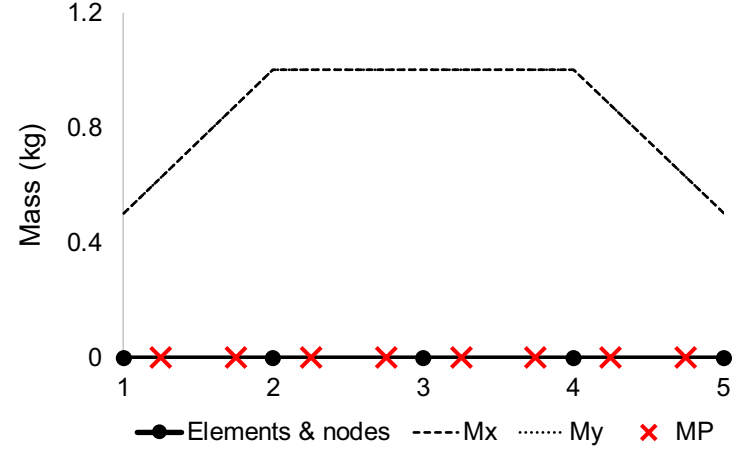

(b)

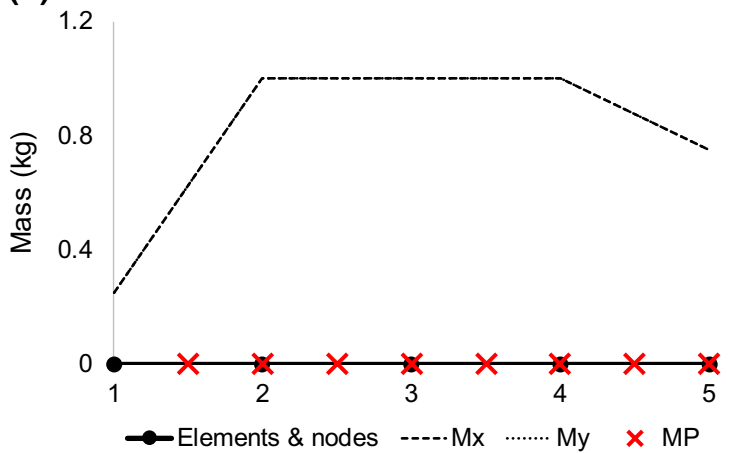

Fig. 13 Nodal mass distribution considering $\mathbf{a}$ initial material point distribution, and $\mathbf{b}$ material point distribution after horizontal movement

point distributions inside an element, but the nodal mass and nodal external forces are the same in both cases.

Figure 13 shows the distribution of $\mathbf{M}$ for the same problem as in Fig. 11. It is clear that the movement of material points and the crossing of nodes does not cause any trouble for the nodal integration because of the continuity of the SFs. Also, since the integration of $\mathbf{F}^{\mathrm{ext}}$ is performed in a similar manner to $\mathbf{M}$, the distribution would be similar to the one in Fig. 13.

\subsection{Plastic stress redistribution}

The stress oscillation caused by the plastic stress redistribution is an extension of the oscillations explained in the previous sections. As the stresses exceeding the yield surface are integrated as a new external force computed with SF gradients, additional oscillations comparable to the $\mathbf{F}^{\text {int }}$ oscillations are introduced. Moreover, oscillating stresses could cause some points to yield spuriously, leading to an unrealistic system behaviour.

\section{Improvements to reduce stress oscillations}

\subsection{GIMP}

The generalised interpolation material point (GIMP) method [6] was proposed to reduce oscillations derived from material points crossing element boundaries. In GIMP, FE SFs are replaced by functions constructed based on the linear FE SF and a material point support domain (SD). This means that each material point has a domain over which its influence is distributed. The GIMP SF $\left(\mathrm{S}_{\mathrm{ip}}\right)$ and its gradient $\left(\nabla \mathrm{S}_{\mathrm{ip}}\right)$ in one dimension are computed as

$\mathrm{S}_{\mathrm{ip}}=\frac{1}{\mathrm{~V}_{\mathrm{p}}} \int_{\Omega_{\mathrm{p}} \cap \Omega} \chi_{\mathrm{p}}(\mathrm{x}) \mathrm{N}_{\mathrm{i}}(\mathrm{x}) \mathrm{dx}$ $\nabla \mathrm{S}_{\mathrm{ip}}=\frac{1}{\mathrm{~V}_{\mathrm{p}}} \int_{\Omega_{\mathrm{p}} \cap \Omega} \chi_{\mathrm{p}}(\mathrm{x}) \nabla \mathrm{N}_{\mathrm{i}}(\mathrm{x}) \mathrm{dx}$

where $\mathrm{V}_{\mathrm{p}}$ is the material point volume, $\Omega$ is the problem domain, $\Omega_{\mathrm{p}}$ is the material point support domain, $\mathrm{i}$ is the node, and $\chi_{\mathrm{p}}$ is the characteristic function delimiting the area of influence of the material point and is given as

$\chi_{\mathrm{p}}(\mathrm{x}) \begin{cases}1, & \text { if } \mathrm{x} \cap \Omega_{\mathrm{p}} \\ 0, & \text { otherwise }\end{cases}$

The support domain is often assumed to be square, with a size of $2 \mathrm{lp}(\mathrm{lp}=$ half of the material point support domain), which is obtained by dividing the element size by the number of material points. In Fig. 14, a 1D comparison between an FE SF and a GIMP SF is plotted, considering a distribution of two equally-distributed material points per element. It is seen that the GIMP SF and GIMP SF gradients are no longer exclusive to a single element and that the GIMP SF gradients are continuous between elements.

The GIMP SFs in 2D and 3D are computed as products of the 1D GIMP SF in each direction; that is, $\mathrm{S}_{\mathrm{i}}(\mathrm{x})=\mathrm{S}_{\mathrm{ip}}^{1}\left(\mathrm{x}_{1}\right) \cdot \mathrm{S}_{\mathrm{ip}}^{2}\left(\mathrm{x}_{2}\right) \quad$ i $\quad 2 \mathrm{D} \quad$ a $\mathrm{n} \mathrm{d}$ $S_{i}(x)=S_{i p}^{1}\left(x_{1}\right) \cdot S_{i p}^{2}\left(x_{2}\right) \cdot S_{i p}^{3}\left(x_{3}\right)$ in $3 D$, where $S_{\text {ip }}^{k}$ is the 1D GIMP SF in the k-direction. An additional advantage of including a support domain is that the material boundary is explicitly defined, and can be used to apply boundary conditions.

\subsection{Modified integration weights}

To reduce the problems caused by an irregular number of material points inside an element, it is here proposed to modify the material point integration weight to

$\mathrm{W}^{*}=\mathrm{W} \frac{\mathrm{omp}}{\mathrm{cmp}}$ 


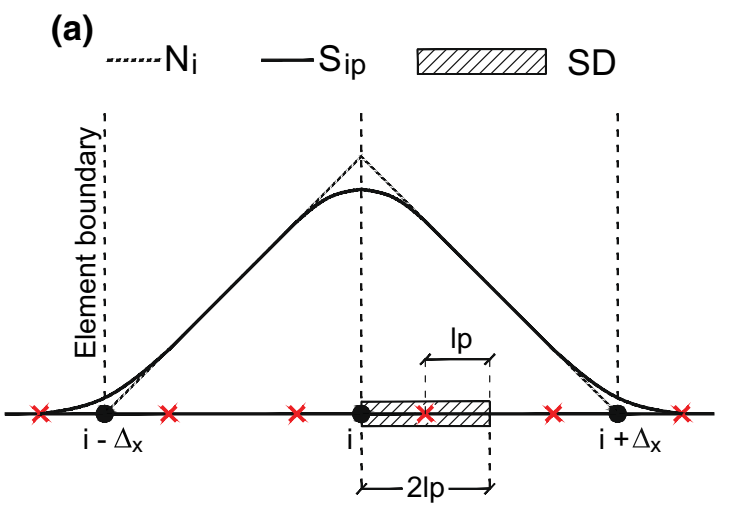

(b)

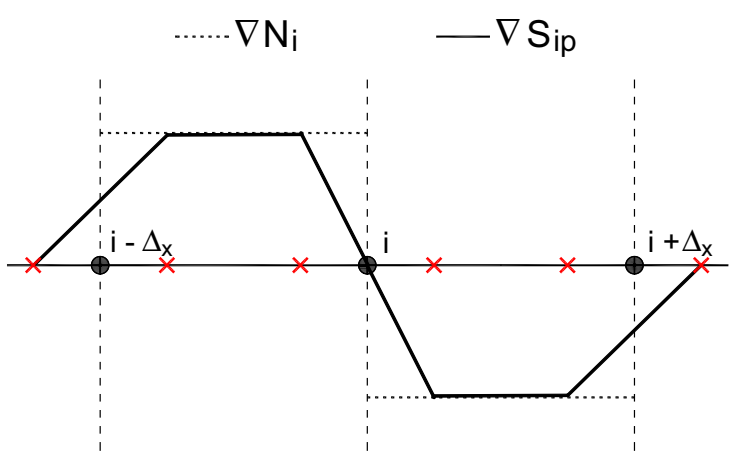

Fig. 14 a GIMP shape function $\left(\mathrm{S}_{\mathrm{ip}}\right)$ and regular FE shape function $\left(\mathrm{N}_{\mathrm{i}}\right)$ of node $\mathrm{i}$, and $\mathbf{b}$ GIMP shape function gradient $\left(\nabla \mathrm{S}_{\mathrm{ip}}\right)$ and regular FE shape function gradient $\left(\nabla \mathrm{N}_{\mathrm{i}}\right)$ for node $\mathrm{i}$

where $\mathrm{W}^{*}$ is the modified material point weight (dimensionless), cmp is the current number of material points in the element, and omp is the original number of material points in the element. This modified weight is used considering only structured meshes, i.e. a mesh composed of equal-sized square elements, and equal mass material points, and its use with unstructured meshes or unequal mass material points is not part of this work. This modified weight technique differs from the approach of other researchers who have modified the weights based on volumetric strain (e.g. [11]), which, while compensating for $1 \mathrm{D}$ deformations of the material points (compression or extension), does not reduce the problems caused by the rotation or advection of the material points. Finally, it should be noted that for four noded elements this modified weight value reduces to $4.0 / \mathrm{cmp}$.

\subsection{Double mapping (DM)}

Integration using SF gradients is seen to work only at Gauss point locations, whereas material point integration is stable when based on SFs. Therefore, mapping to the Gauss point locations using shape functions (via the nodes) is proposed. As an example, the stiffness matrix is used. The elastic matrix is mapped to the nodes from the material points and then to the Gauss points, prior to the integration. Using FE SFs, the material point elastic matrix is mapped to the element nodes as

$\mathbf{D}_{\mathrm{i}}=\sum_{\mathrm{p}=1}^{\mathrm{cmp}} \mathrm{N}_{\mathrm{i}}\left(\mathbf{x}_{\mathrm{p}}\right) \mathbf{D}_{\mathrm{p}} \mathrm{W}^{*}$

were $\mathbf{D}_{\mathrm{i}}$ is the elastic matrix at node $\mathrm{i}$, and $\mathbf{D}_{\mathrm{p}}$ is the elastic matrix of material point $\mathrm{p}$.

At this point, the total stiffness contribution of the material points is accumulated at the nodes, and this contribution is then redistributed to the original Gauss positions as
$\mathbf{D}_{\mathrm{g}}=\sum_{\mathrm{i}=1}^{\mathrm{nn}} \mathrm{N}_{\mathrm{i}}\left(\mathbf{x}_{\mathrm{g}}\right) \mathbf{D}_{\mathrm{i}}$

were $\mathbf{D}_{\mathrm{g}}$ is the elastic matrix at the Gauss point, $\mathrm{N}_{\mathrm{i}}\left(\mathbf{x}_{\mathrm{g}}\right)$ is the nodal SF evaluated at the Gauss points, and $\mathrm{nn}$ is the number of nodes of the element. By substituting Eq. 16 into Eq. 17, $\mathbf{D}_{\mathrm{g}}$ is obtained as

$\mathbf{D}_{\mathrm{g}}=\sum_{\mathrm{i}=1}^{\mathrm{nn}}\left(\mathrm{N}_{\mathrm{i}}\left(\mathbf{x}_{\mathrm{g}}\right) \sum_{\mathrm{p}=1}^{\mathrm{cmp}} \mathrm{N}_{\mathrm{i}}\left(\mathbf{x}_{\mathrm{p}}\right) \mathbf{D}_{\mathrm{p}} \mathrm{W}^{*}\right)$

Finally, combining Eqs. 18 and 5 (in FEM form) results in the nodal stiffness:

$\mathbf{K}=\sum_{\mathrm{g}=1}^{\text {ngauss }} \mathbf{B}^{\mathrm{T}}\left(\mathbf{x}_{\mathrm{g}}\right)\left[\sum_{\mathrm{i}=1}^{\mathrm{nn}}\left(\mathrm{N}_{\mathrm{i}}\left(\mathbf{x}_{\mathrm{g}}\right) \sum_{\mathrm{p}=1}^{\mathrm{cmp}} \mathrm{N}_{\mathrm{i}}\left(\mathbf{x}_{\mathrm{p}}\right) \mathbf{D}_{\mathrm{p}} \mathrm{W}^{*}\right)\right] \mathbf{B}\left(\mathbf{x}_{\mathrm{g}}\right)|\mathbf{J}| \mathrm{W}^{\mathrm{FE}}$

where ngauss is the number of Gauss points in the element and $\mathrm{W}^{\mathrm{FE}}$ is the weight associated with Gauss point $\mathrm{g}$ (as in FEM).

\subsection{DM-GIMP (DM-G)}

As mentioned in Sect. 5.1, the GIMP method was created to avoid problems caused by the use of discontinuous FE $\mathrm{SF}$ gradients. However, a simple example in calculating the stiffness reveals a key problem. Figure 15 shows the same problem as in Fig. 11, but in this case the stiffness is computed using regular SFs and GIMP SF gradients.

As shown in Fig. 15a, for the initial configuration of material points, the nodal stiffness distributions remain the same for both techniques, because at this position the MPM and GIMP SFs and SF gradients are the same. With the movement of the material points (Fig. 15b), the nodal stiffness computed with GIMP decreases, as the GIMP SF gradients drop to zero at the inter-element boundaries (as shown 
(a)

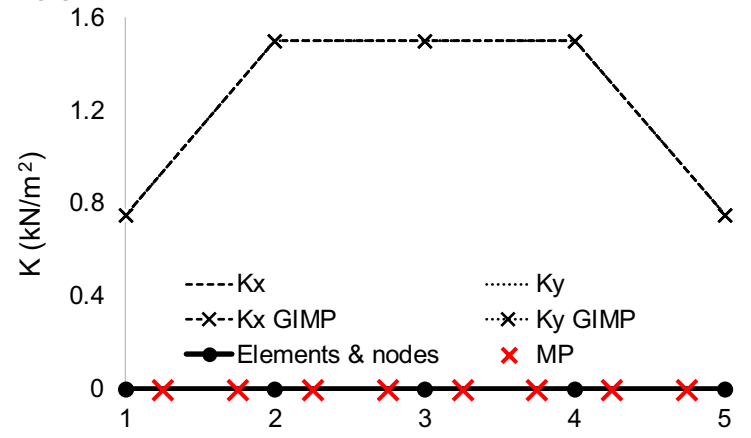

(b)

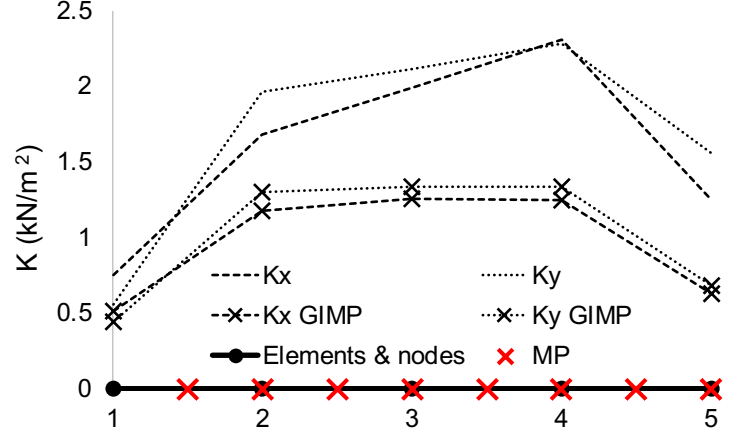

Fig. 15 Nodal stiffness computed using regular SFs and GIMP SFs considering a initial material point positions, and $\mathbf{b}$ after displacement of material points

Fig. 16 a Nodal FE SF and interaction with the material point support domain, $\mathbf{b}$ original GIMP SF $\left(S_{\text {ip }}\right)$, $\mathbf{c}$ nodal FE SF and interaction with the material point support domain in a single element, and $\mathbf{d}$ local $\operatorname{GIMP} \mathrm{SF}\left(\mathrm{S}_{\mathrm{ip} *}\right)$ (a)

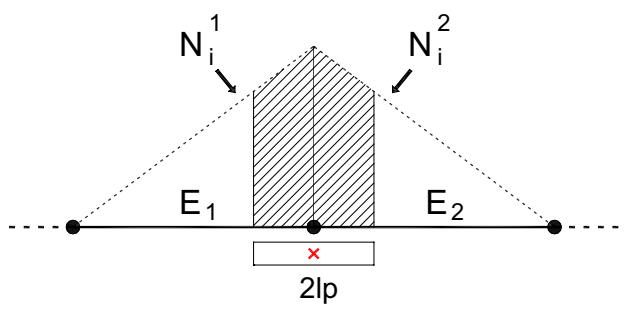

(b)

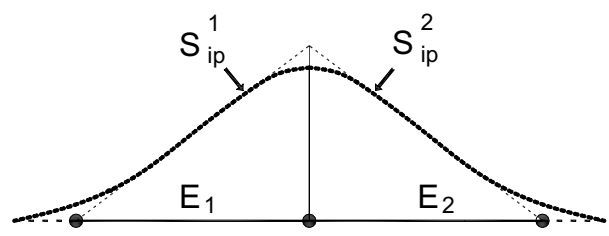

(c)

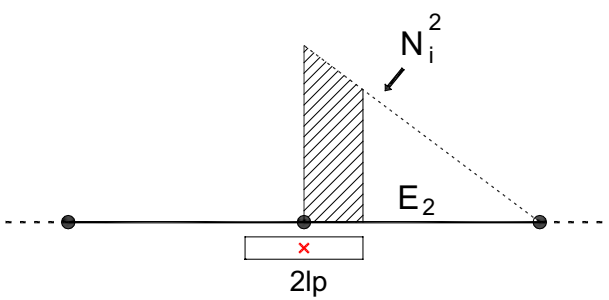

(d)

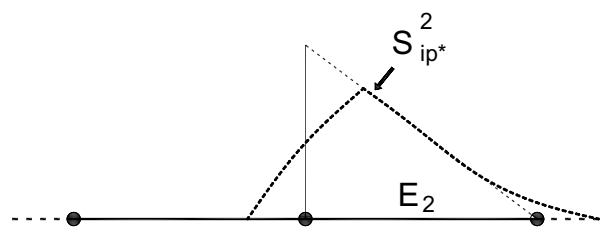

in Fig. 14). In addition, the contribution of material points in neighbouring elements is not capable of compensating for this drop. This would be the case for other methods, including DDMP and CDPI, that have this same characteristic.

To overcome the problems of using GIMP to integrate nodal stiffness, it has been proposed that the double mapping approach be used alongside the local GIMP SFs [10]. The local GIMP SFs $\left(\mathrm{S}_{\mathrm{ip} *}\right)$ are similarly created as regular GIMP SFs, but the influence of the material point support domain affects only the nodal FE SF in a single element rather than contributing to all contiguous elements. In Fig. 16, an illustration of the development of regular and local GIMP shape functions of a node is shown.

In a similar manner to the double mapping technique using regular SFs, by using local GIMP SFs it is possible to distribute the elastic matrix to the nodes of an element and afterwards to the Gauss positions. The element stiffness matrix is then constructed as
$\mathbf{K}_{\mathrm{el}}=\sum_{\mathrm{g}=1}^{\text {ngauss }} \mathbf{B}^{\mathrm{T}}\left(\mathbf{x}_{\mathrm{g}}\right)\left[\sum_{\mathrm{i}=1}^{\mathrm{nn}}\left(\mathrm{N}_{\mathrm{i}}\left(\mathbf{x}_{\mathrm{g}}\right) \sum_{\mathrm{p}=1}^{\mathrm{smp}} \mathrm{S}_{\mathrm{ip} *}\left(\mathbf{x}_{\mathrm{p}}\right) \mathbf{D}_{\mathrm{p}} \mathrm{W}\right)\right] \mathbf{B}\left(\mathbf{x}_{\mathrm{g}}\right)|\mathbf{J}| \mathrm{W}^{\mathrm{FE}}$

where $\mathrm{S}_{\mathrm{ip}}$ is the local GIMP SF of node $\mathrm{i}$ evaluated at the material point position, and smp is the number of material points with a support domain inside the element. The algorithm to compute the stiffness matrix using DM and DM-G is given in "Appendix C", together with a study of the computational performance.

\subsection{Composite material point method (CMPM)}

The composite material point method (CMPM) [14] is a modification of the composite finite element method (CFEM), proposed by Sadeghirad \& Astaneh [23], in which the support domain used to recover the stresses is extended, i.e. a patch, improving the accuracy of the stresses computed. New shape 


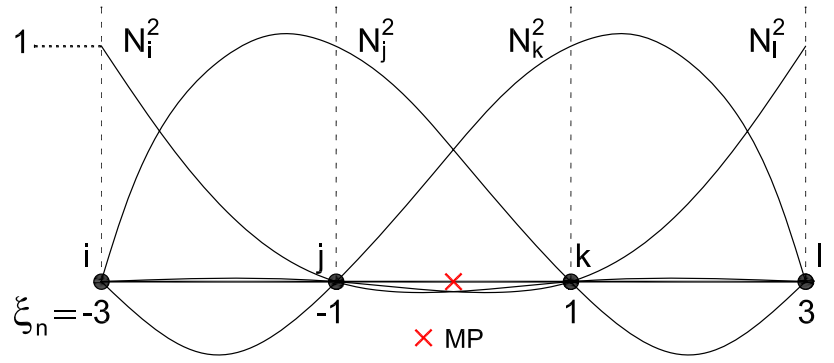

Fig. 17 CMPM shape functions with $\mathrm{C}^{2}$ continuity for a central local element

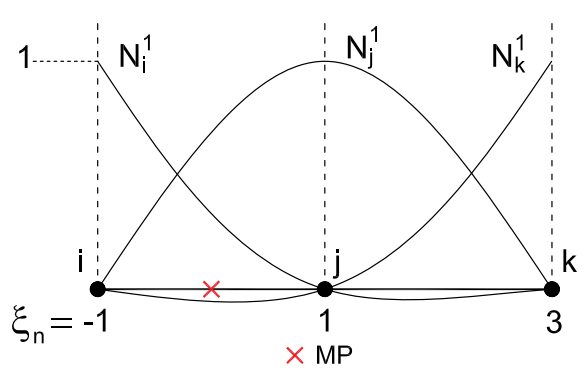

Fig. 18 CMPM shape function with $\mathrm{C}^{1}$ continuity for a boundary local element

functions enveloping all neighbouring elements of the element containing the material point are developed using Lagrange interpolation. In Fig. 17, the $\mathrm{C}^{2}$ shape functions are shown in $1 \mathrm{D}$, in which each shape function $\mathrm{N}^{2}$ envelopes the local element plus the neighbouring elements.

Using Lagrange interpolation, each of the $\mathrm{N}^{2}$ shape functions is computed as

$$
\mathrm{N}_{\mathrm{n}}^{2}(\xi)=\prod_{\substack{\mathrm{m}=1 \\ \mathrm{~m} \neq \mathrm{j}}}^{\mathrm{n}} \frac{\xi-\xi_{\mathrm{m}}}{\xi_{\mathrm{j}}-\xi_{\mathrm{m}}}
$$

where $\xi$ is the nodal local coordinate in the extended domain, $\mathrm{n}$ is the number of nodes, $\xi_{\mathrm{j}}$ is the local coordinate of the $\mathrm{N}_{\mathrm{i}}^{2}$ shape function, and $\xi_{\mathrm{m}}$ is the local coordinate of the remaining nodes. Solving Eq. 21 for each node, the CMPM shape functions for an element with two neighbours are

$\left[\begin{array}{c}N_{i}^{2} \\ N_{j}^{2} \\ N_{k}^{2} \\ N_{1}^{2}\end{array}\right]=\frac{1}{16}\left[\begin{array}{c}-\frac{\xi^{3}-3 \xi^{2}-\xi+3}{3} \\ \xi^{3}-\xi^{2}-9 \xi+9 \\ -\xi^{3}-\xi^{2}+9 \xi+9 \\ \frac{\xi^{3}+3 \xi^{2}-\xi-3}{3}\end{array}\right]$

If the material point is located at the boundary, as in Fig. 18, the CMPM shape functions are then

$\left[\begin{array}{c}\mathrm{N}_{\mathrm{i}}^{1} \\ \mathrm{~N}_{\mathrm{j}}^{1} \\ \mathrm{~N}_{\mathrm{k}}^{1}\end{array}\right]=\frac{1}{4}\left[\begin{array}{c}\frac{\xi^{2}-4 \xi+3}{2} \\ -\xi^{2}+2 \xi+3 \\ \frac{\xi^{2}+4 \xi+3}{2}\end{array}\right]$

It is important to mention that although the CMPM SFs extend beyond the limits of an element, the range of the functions remains between $-1 \leq \xi \leq 1$. Also, this solution can only be used with a structured mesh. To extend the solution to a 2D domain, the new SFs are the product of the SFs in each direction. Finally, trial stresses using CMPM are computed as

$\Delta \boldsymbol{\sigma}_{\mathrm{p}}=\mathbf{D}_{\mathrm{p}} \nabla \mathbf{N}^{2}\left(\mathbf{x}_{\mathrm{p}}\right) \Delta \overline{\mathbf{u}}^{\mathrm{ext}}$

where $\nabla \mathbf{N}^{2}$ is the matrix of the CMPM SF gradients, and $\overline{\mathbf{u}}^{\mathrm{ext}}$ is the vector of nodal displacements in the extended domain.

\section{Testing of proposed techniques}

Since the novel techniques presented in this paper are designed for the integration of the stiffness, the testing performed in this section is focused on the stiffness matrix. To compare the stiffness using each technique, the stiffness magnitude is used, and this is computed as

$\mathrm{K}_{\mathrm{mag}}=\sqrt{\mathrm{K}_{\mathrm{x}}^{2}+\mathrm{K}_{\mathrm{y}}^{2}}$
Fig. 19 Infinite domain full of equally spaced material points a before and $\mathbf{b}$ after rotation (a)

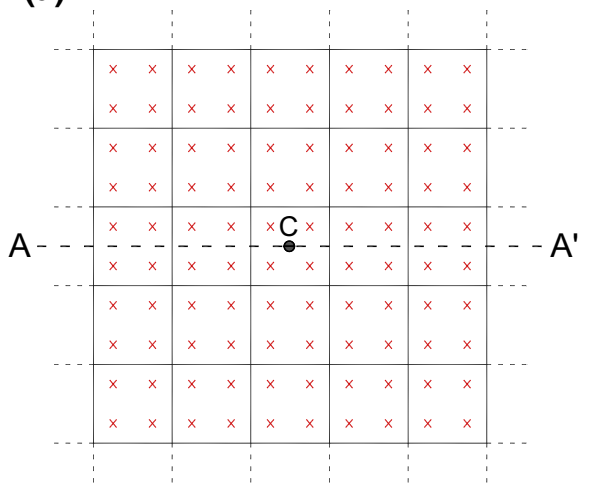

(b)

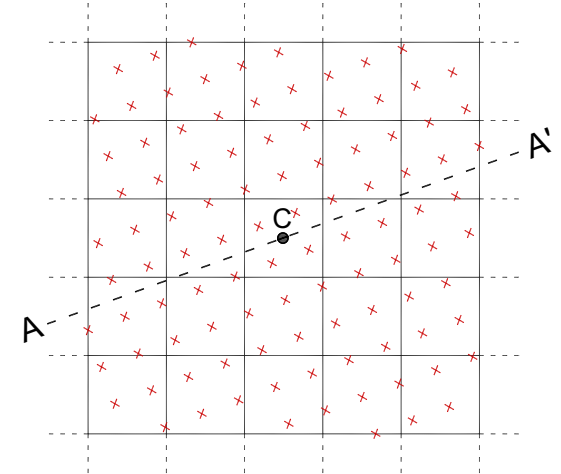


Fig. 20 Stiffness distribution considering rotation of the domain, using one and two materials, computed with FEM, MPM, W*, GM and DM
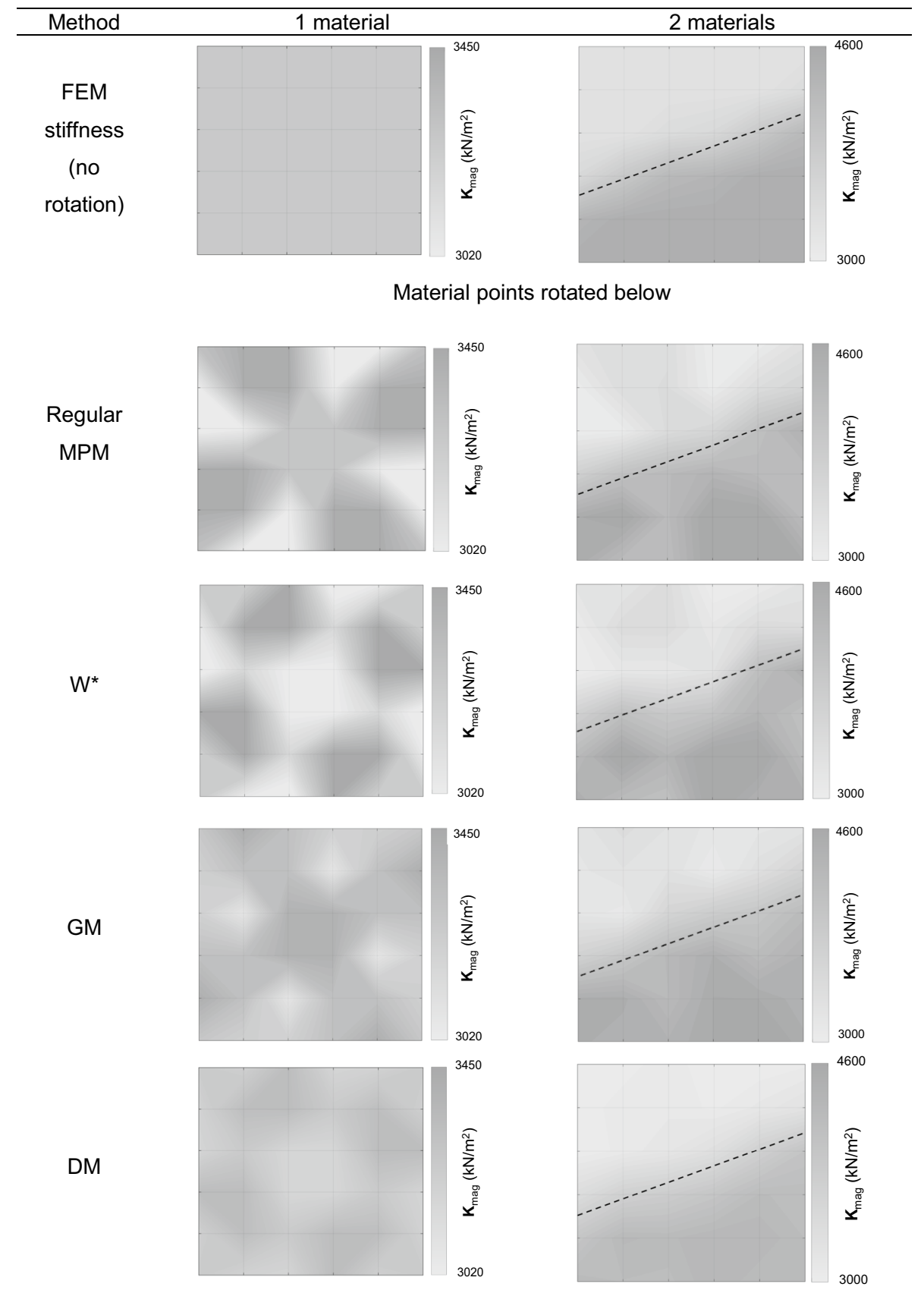

The test consists of computing the stiffness of an infinite space made up of square elements that are full of equally spaced material points, four per element, as shown in Fig. 19a. The infinite domain is then rotated $20^{\circ}$ degrees around its centre (C), as in Fig. 19b. The elastic properties of the material are $\mathrm{E}=1000 \mathrm{kN} / \mathrm{m}^{2}$ and $\nu=0.30$. Figure 20 presents the stiffness computed using regular MPM and DM and the results are compared with the FEM stiffness, computed using four Gauss integration points $\left(\mathrm{K}_{\mathrm{mag}}=3263.57 \mathrm{kN} /\right.$ $\mathrm{m}^{2}$ ). In addition, the stiffness using the modified integration weights $\left(\mathrm{W}^{*}\right)$ and Gauss mapping (GM) separately (the two components of DM) are shown to highlight their comparative effects. Since the material points remain equally distributed after rotation, the stiffness of the domain should not change (i.e. be mesh independent). Finally, a further test is performed using two materials, by considering the properties of material points below line $\mathrm{A}-\mathrm{A}^{\prime}$ to be $\mathrm{E}=1500 \mathrm{kN} /$ $\mathrm{m}^{2}$ and $\nu=0.25$.

Theoretically, the stiffness of the domain should be independent of the rotation of the field of material points, and should be equal to the FEM stiffness before rotation (for the case with one material). As can be observed in Fig. 20, the stiffness obtained using regular MPM is not accurate and improvements are needed. After including the modified 
integration weight $\left(\mathrm{W}^{*}\right)$, which accounts for a varying amount of material points per element, the stiffness distribution oscillates, although with a different spatial pattern than in regular MPM. Using GM the oscillation also persists, as the number of material points per cell is still incorrect, but it is less than in regular MPM because it helps to reduce errors due to material point position. It is noted that including $\mathrm{W}^{*}$ or GM separately is unable to fix the stiffness oscillation, and that the spatial distribution is almost opposite in pattern, i.e. where high values occur in $\mathrm{GM}$, low values occur in $\mathrm{W}^{*}$, and vice versa. Using DM, i.e. combining GM and $\mathrm{W}^{*}$, the stiffness oscillation is reduced significantly, as it accounts for both the material point position and the number of material points per element. Moreover, the transition is smooth over the elements when two materials are used.

In Fig. 21, the tests from Fig. 19 have been performed using GIMP and DM-G. As can be observed, the stiffness obtained using GIMP integration is significantly more inaccurate when compared to MPM integration, as it both oscillates and reduces in magnitude. Note that the results for GIMP are shown using a different contour range; this is because using GIMP SF gradients the stiffness reduces significantly, and it is necessary to change the contour range to visualize the stiffness distribution. On the other hand, using DM-G the stiffness oscillation is reduced further than using DM. This is because the $\mathrm{W}^{*}$ approach, which only allows the impact of a discrete number of points in each element to be considered, is not being used. Utilising DM-G allows a gradual transition of mass from one element to another. Moreover, using DM-G, the transitions between materials appears sharper than in regular FEM due to an increase in the accuracy of the material stiffness distribution between the interface nodes.

In Table 1, the difference between the stiffness obtained using each technique is shown relative to the nodal stiffness magnitude of the real FEM stiffness. In this comparison,
Table 1 Relative differences in stiffness magnitude between FEM and other methods for homogeneous material

\begin{tabular}{llc}
\hline Method & Maximum increase (\%) & Maximum decrease (\%) \\
\hline Regular MPM & 5.51 & -7.38 \\
W $^{*}$ & 5.93 & -8.88 \\
GM & 4.75 & -5.63 \\
DM & 2.23 & -2.38 \\
GIMP $^{\mathrm{a}}$ & 0.0 & -33.95 \\
DM-G & 0.21 & -0.39 \\
\hline
\end{tabular}

${ }^{\mathrm{a}}$ Using GIMP the stiffness only decreases with respect to FEM

only the homogenous material is considered. As can be observed, regular MPM and GIMP give large stiffness oscillations relative to the FEM stiffness, but in the case of GIMP the stiffness only decreases (as observed also in Fig. 15b). Using only the modified integration weight the stiffness oscillation increases, whereas using the GM stiffness the oscillation decreases (compared to regular MPM), but not significantly. Using DM and DM-G, the dependence between the mesh and the position of the material points is reduced, and the nodal stiffness oscillations reduce significantly, especially using DM-G where the variation is smaller than $1 \%$.

\section{Benchmark problems including improvements}

The benchmark problems introduced in Sect. 3 are now reanalysed using the improvements described in Sect. 5.
Fig. 21 Stiffness distribution considering rotation of the domain, using one and two materials, computed with GIMP and DM-G

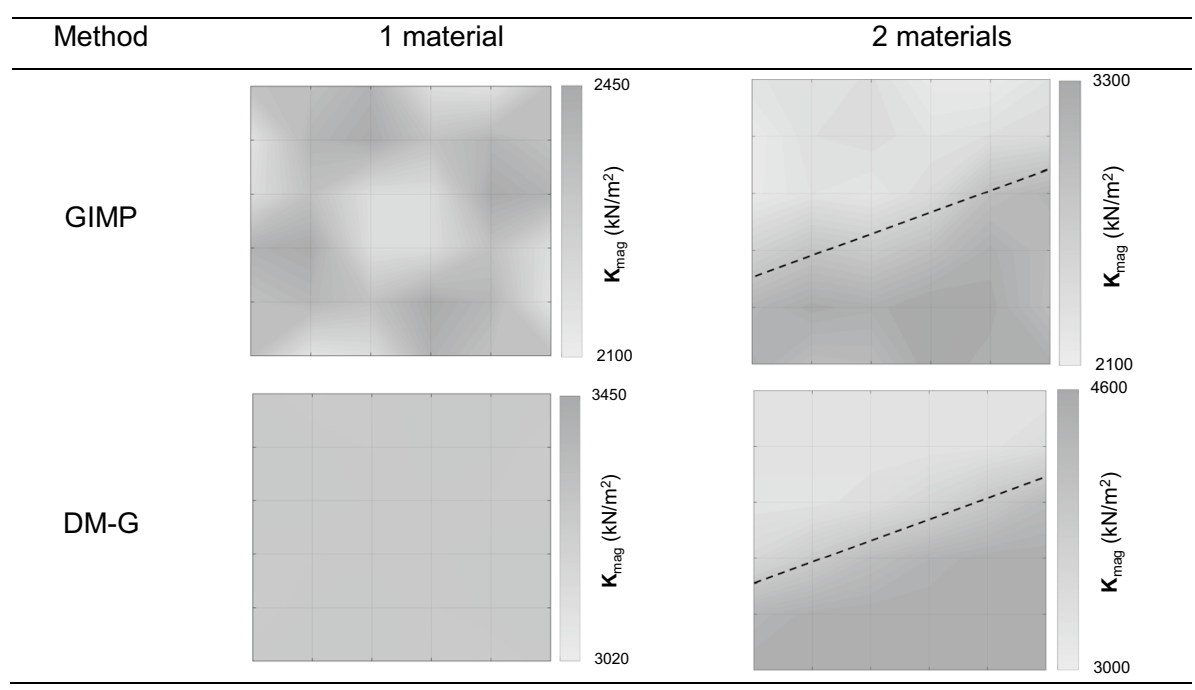




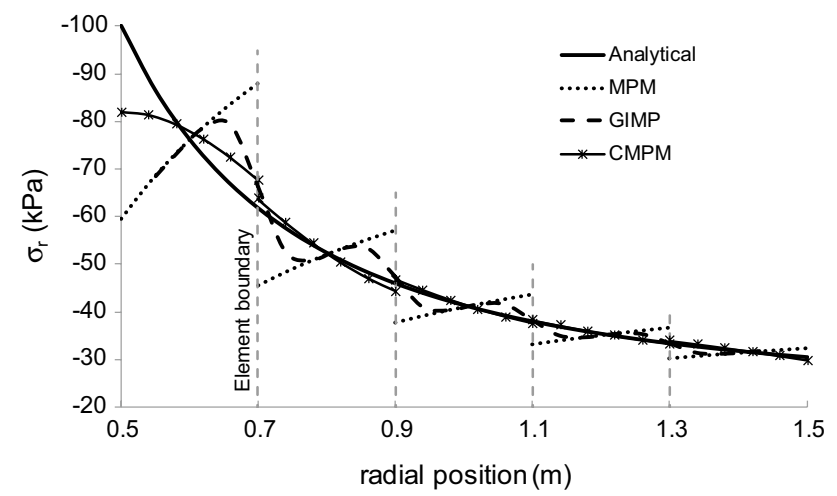

Fig. 22 Analytical, MPM, GIMP and CMPM radial stresses through the cylinder wall

\subsection{Axisymmetric benchmark}

Figure 8 showed the stress oscillation caused by using regular SFs to recover stresses in the cylinder wall. In Fig. 22, GIMP and CMPM are compared against regular MPM for a single (i.e. the first) load step. As can be seen, the GIMP
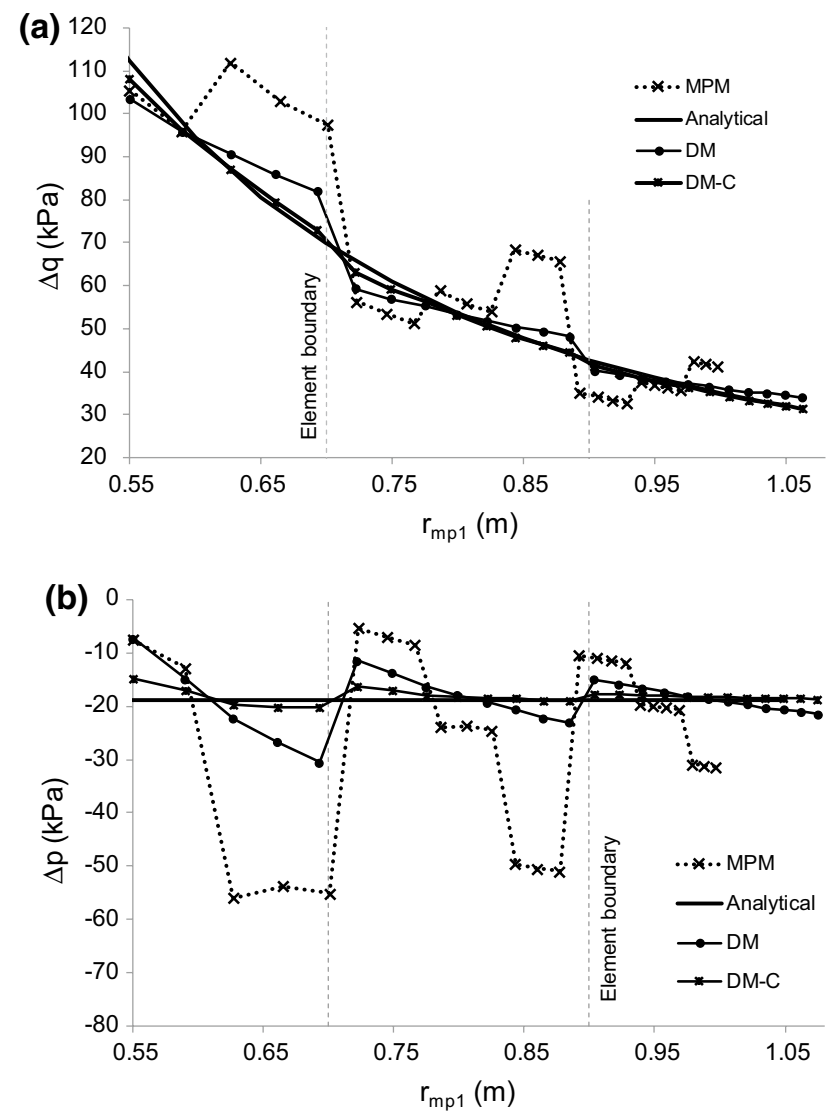

Fig. 23 a Deviatoric, and $\mathbf{b}$ mean stress recovered from $\mathrm{mp}_{1}$ at different positions using DM and DM-C oscillation is the same as MPM close to the centre of the element, because there the SF gradients are the same for both techniques. However, stresses are continuous between the elements, due to the continuous gradients of GIMP. On the other hand, using CMPM the stresses remain discontinuous between elements, but the reduction of oscillation when compared to the analytical solution is significant.

In Fig. 23, the evolution of the incremental deviatoric and mean stresses of material point $\mathrm{mp}_{1}$ (over 25 load steps) are shown, comparing the stresses obtained using normal MPM (as shown in Fig. 4), DM and DM-CMPM (DM-C). As can

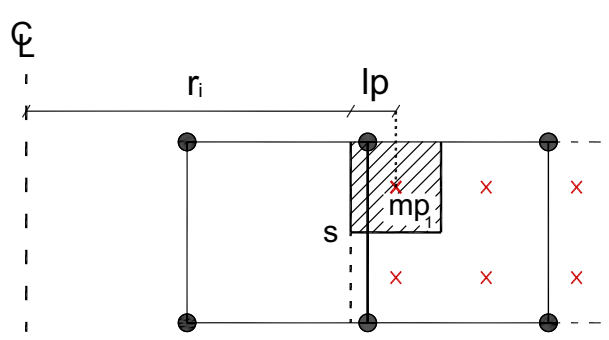

Fig. 24 Internal boundary location at a given step using the GIMP support domain
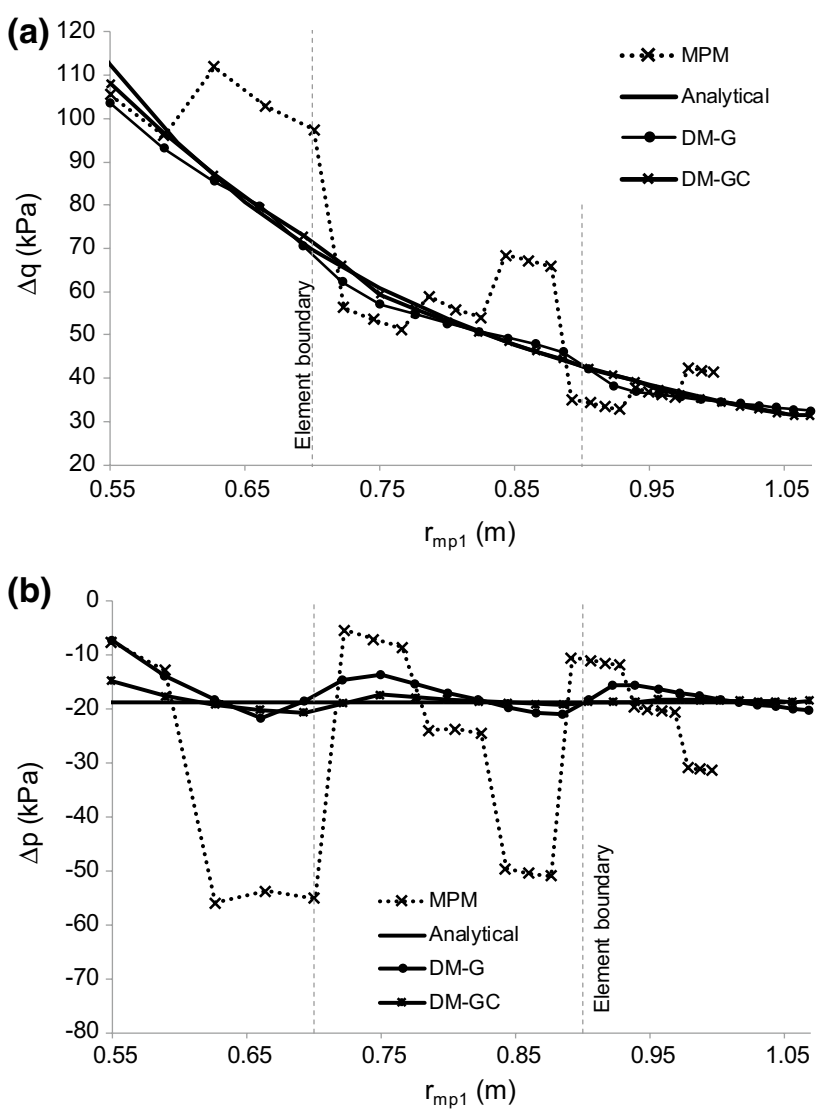

Fig. 25 a Deviatoric, and $\mathbf{b}$ mean stress recovered from $\mathrm{mp}_{1}$ at different positions using DM-G and DM-GC 
be seen, there is a significant increase in the accuracy of the stresses recovered using the DM technique, due to the improved stress recovery and stiffness integration. Moreover, if CMPM is included in the analysis, the stress oscillation reduces still further to give stresses close to the analytical solution.

Next, the same example using DM-G and DM-GIMPCMPM (DM-GC) is studied. Using DM-G, the stiffness is computed with the DM-G method and the stresses are recovered using GIMP SFs. Using DM-GC, the DM-G method is again used to compute the stiffness, but the stresses are now recovered using CMPM rather than GIMP. In addition, since the inner wall boundary can be determined accurately using the material point support domain (as mentioned in Sect. 5.1), the distance between the cylinder axis and the inner boundary (s) is $r_{i}=r_{m p 1}-l p$ as in Fig. 24. Then, the applied pressure $\Delta \mathrm{p}_{\mathrm{s}}$ is distributed linearly to the nodes of the boundary element based on proximity.

In Fig. 25 it can be seen that, using DM-G and DM-GC, the results approximate the analytical solution even better than DM and DM-C, respectively. This is because the stiffness computed using DM-G is closer to the FEM stiffness and also due to the accurate distribution of the external pressure considering the accurate location of the internal boundary. (a)

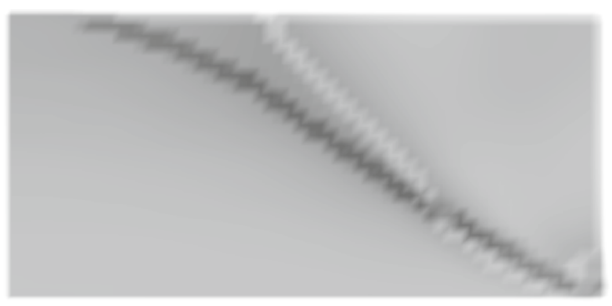

(b)

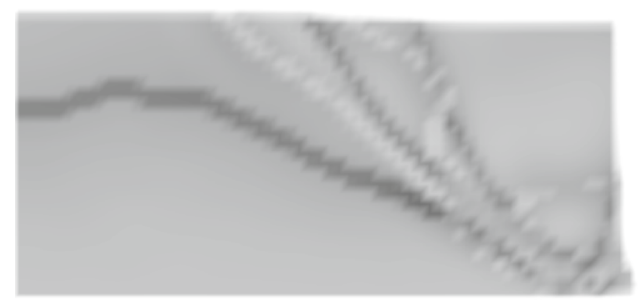

(c)

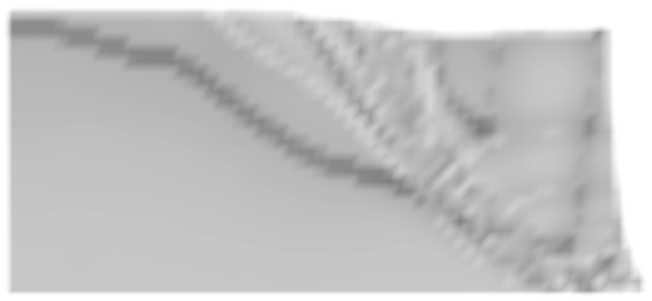

(d)

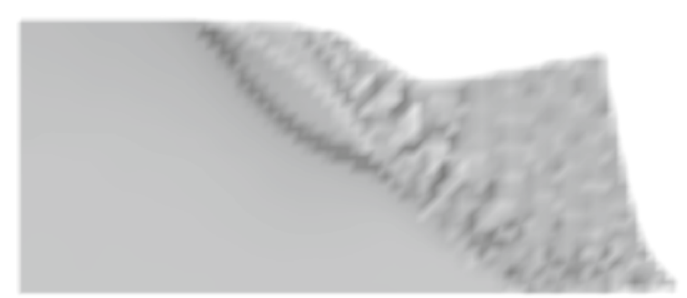

(e)

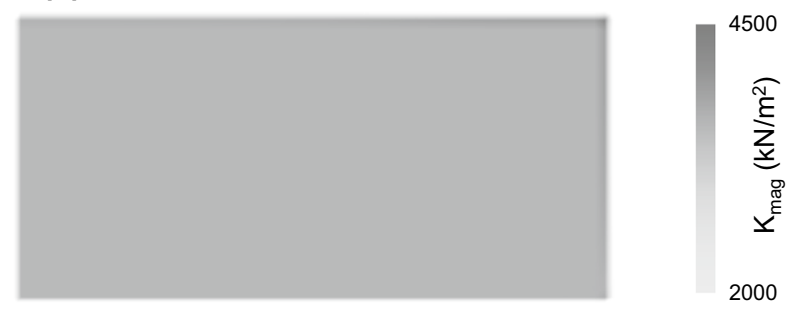

(f)

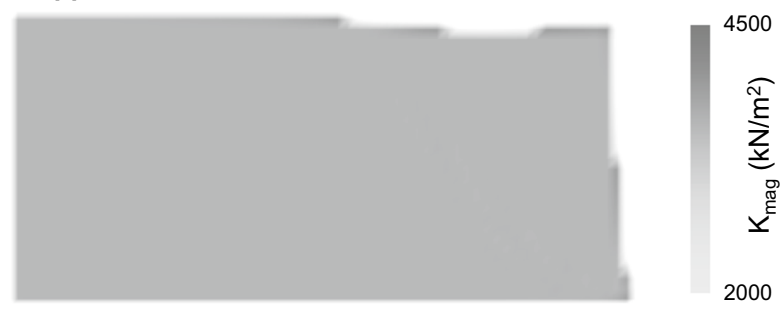

(g)

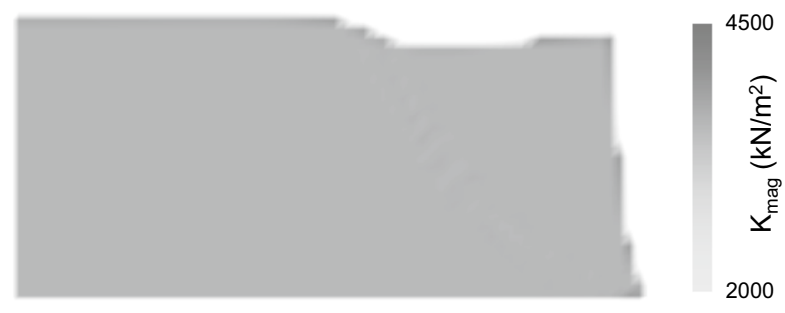

(h)

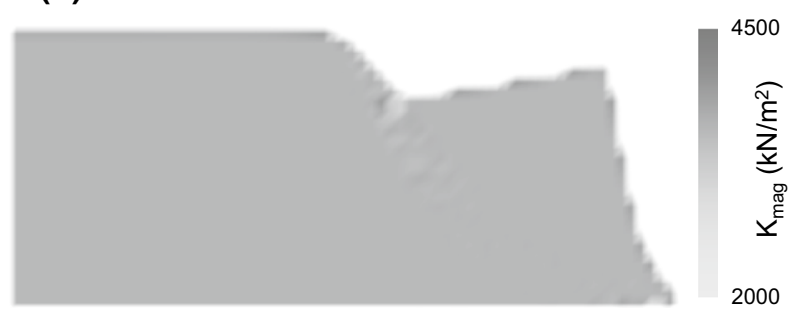

Fig. 26 Stiffness magnitude in the body using regular MPM (a-d) and DM-GC (e-h) after a horizontal toe displacement of $\mathbf{a} \& \mathbf{e} 0.10 \mathrm{~m}, \mathbf{b} \& \mathbf{f}$ $0.30 \mathrm{~m}, \mathbf{c} \& \mathbf{g ~} 0.50 \mathrm{~m}$, and $\mathbf{d} \& \mathbf{h} 1.0 \mathrm{~m}$ 
(a)

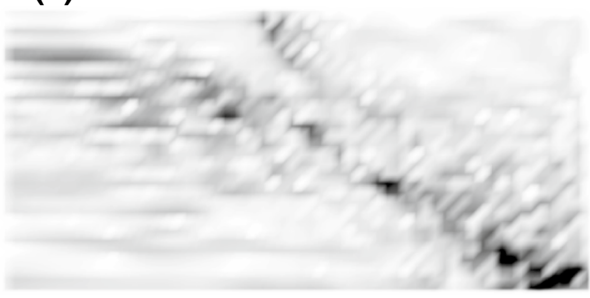

(b)

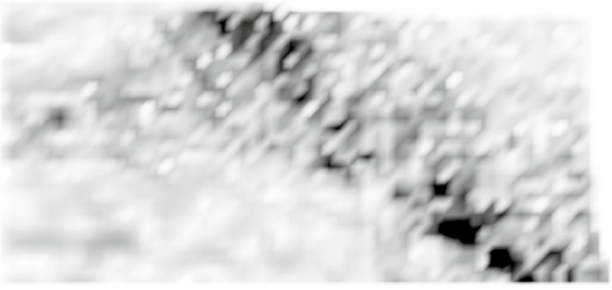

(c)

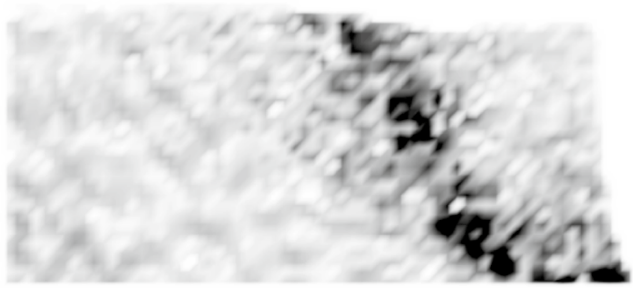

(d)

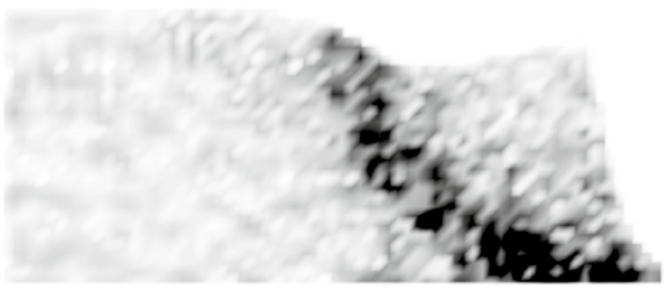

(e)

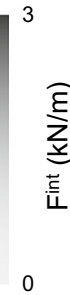

(f)

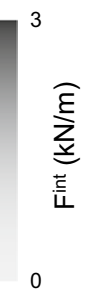

(g)

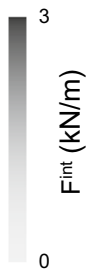

(h)

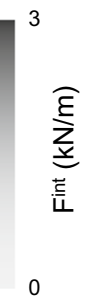

Fig. $27 \mathbf{F}^{\text {int }}$ magnitude in the body using regular MPM (a-d) and DM-GC (e-h) after a horizontal toe displacement of a \& e $0.10 \mathrm{~m}, \mathbf{b} \& \mathbf{f}$ $0.30 \mathrm{~m}, \mathbf{c} \& \mathbf{g} 0.50 \mathrm{~m}$, and $\mathbf{d} \& \mathbf{h} 1.0 \mathrm{~m}$

\subsection{Vertical cut benchmark}

Figure 26 shows the elastic stiffness magnitude in the vertical cut benchmark problem, using regular MPM and DM-GC. As can be observed in Fig. 26a-d, using regular MPM large stiffness oscillations occur, from the beginning (small deformations) up until the end (large deformations) of the analysis. In contrast, using DM-GC (Fig. 26e-h) the stiffness oscillation reduces significantly, although some small oscillation can be observed in the shear band and along the edge of the domain.

In Fig. 27 the nodal $\mathbf{F}^{\text {int }}$ magnitude is shown, once again comparing regular MPM and DM-GC. Analogous to Eq. 25, the magnitude of the nodal internal force is computed as
$\mathrm{F}_{\mathrm{mag}}^{\mathrm{int}}=\sqrt{\mathrm{F}_{\mathrm{x}}^{\mathrm{int}}+\mathrm{F}_{\mathrm{y}}^{\mathrm{int}}}$

It is seen that if GIMP and CMPM are included in the solution, a large reduction in the oscillations of $\mathbf{F}^{\text {int }}$ is obtained. Using GIMP, the oscillation caused by the material points crossing cell boundaries is reduced. Furthermore, by including CMPM, the recovered stresses are improved, reducing the oscillation caused by the stress recovery position.

Figure 28 shows the deviatoric stress contours from both analyses. It is evident that, after reducing the oscillation in the stiffness and the internal nodal forces by using DM-GC, the deviatoric stress distribution in the domain is significantly smoother. Similarly, Fig. 29 shows the comparison of mean stresses during the analyses, demonstrating that the 
(a)

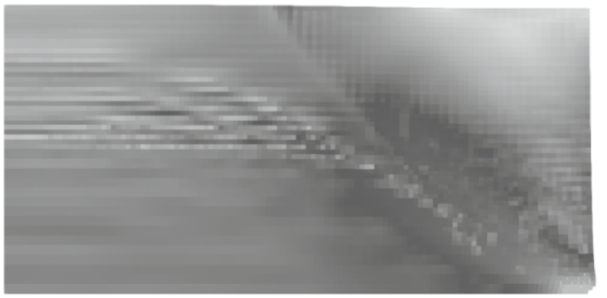

(b)

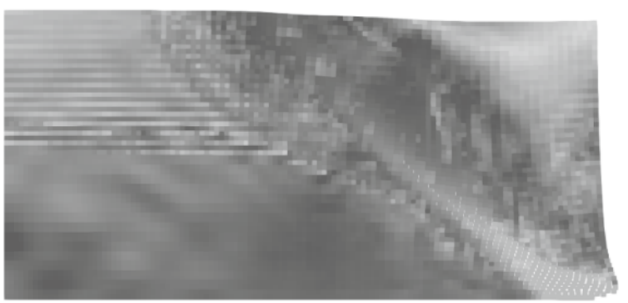

(c)

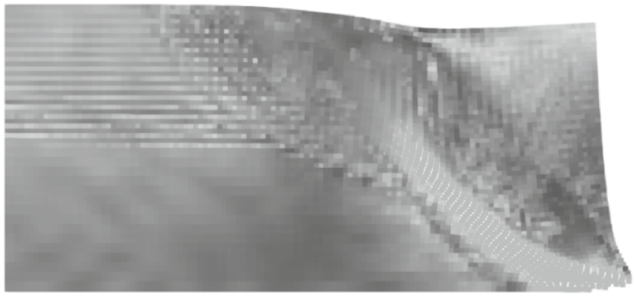

(d)

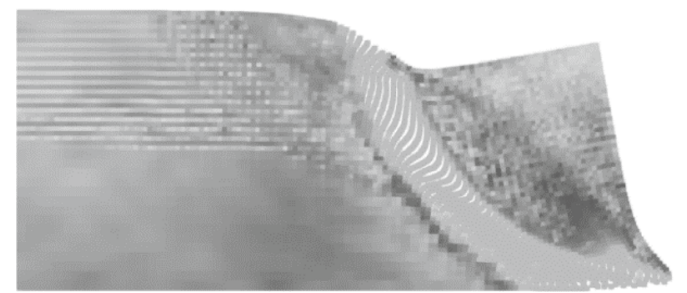

(e)

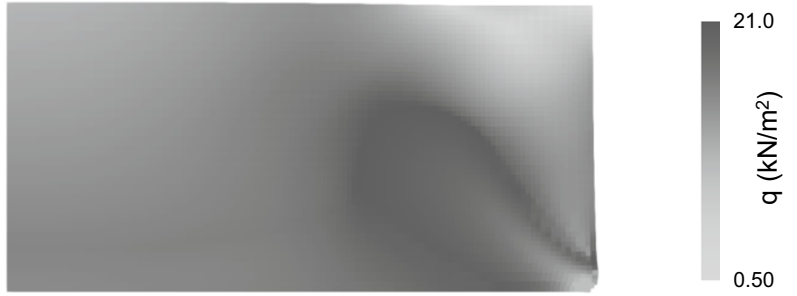

(f)

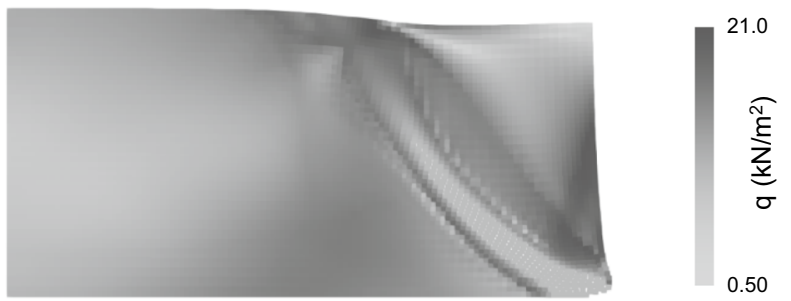

(g)

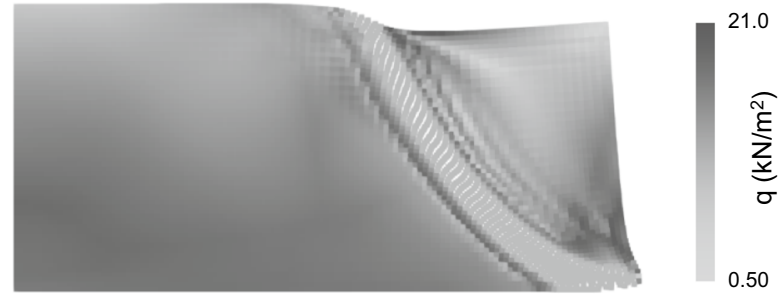

(h)

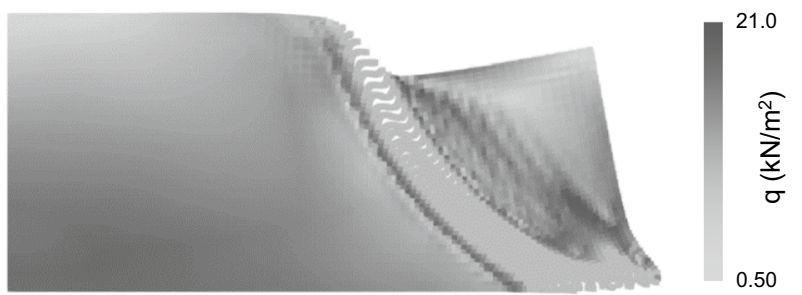

Fig. 28 Deviatoric stresses in the body using regular MPM (a-d) and DM-GC (e-h) after a horizontal toe displacement of a \& e $0.10 \mathrm{~m}, \mathbf{b} \& \mathbf{f}$ $0.30 \mathrm{~m}, \mathbf{c} \& \mathbf{g} 0.50 \mathrm{~m}$, and $\mathbf{d} \& \mathbf{h} 1.0 \mathrm{~m}$

mean stress oscillations are reduced with DM-GC. In this case, as in the axisymmetric benchmark, some oscillation of the mean stresses still occurs, but this is thought to be due to incompressibility during plastic yielding. For methods to reduce locking behaviour in MPM using low order shape functions the reader is referred to Coombs et al. [13].

As can be seen from previous figures, the oscillation of material point stresses, nodal stiffness and internal nodal forces are reduced significantly using DM-GC. Plots for the nodal mass and external nodal forces are not included in the results, since the oscillation for both MPM and DM-GC is small.
Finally, p-q curves have been plotted for 3 material points at key positions in the soil body. Figure 30 shows the location of the points chosen; material point $\mathrm{A}$ is located at the toe of the cutting, material point $\mathrm{B}$ is found in the middle of the soil layer in the shear band, and material point $\mathrm{C}$ is in the centre of the sliding block.

Figure 31 shows the p-q stress paths at the 3 points, as computed using both techniques, as well as the initial position of the yield surface for a Von Mises material $\left(\mathrm{F}_{\mathrm{VM}}\right)$. It is seen that, for material point $\mathrm{A}$, both techniques give reasonable results; this is because the bottom of the domain is fully fixed, so that the material point does not move much throughout the analysis. For material points B and 
(a)

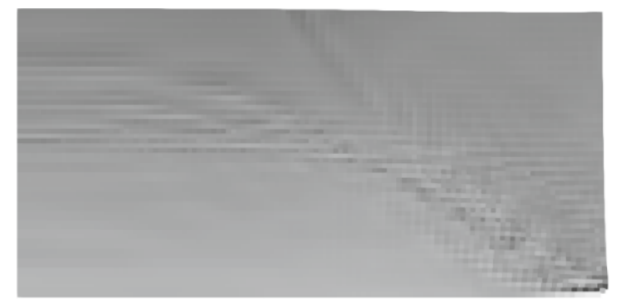

(b)

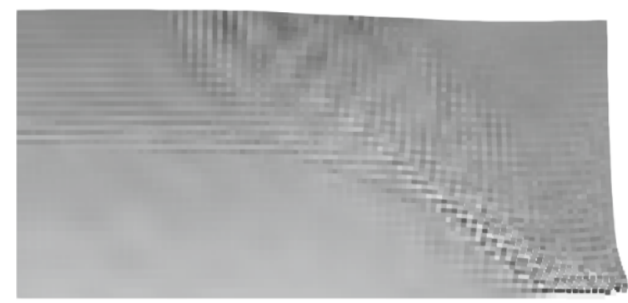

(c)

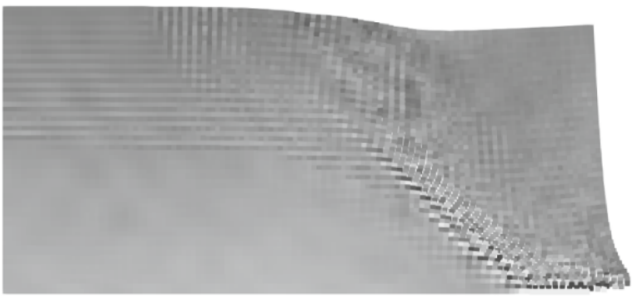

(d) (e)

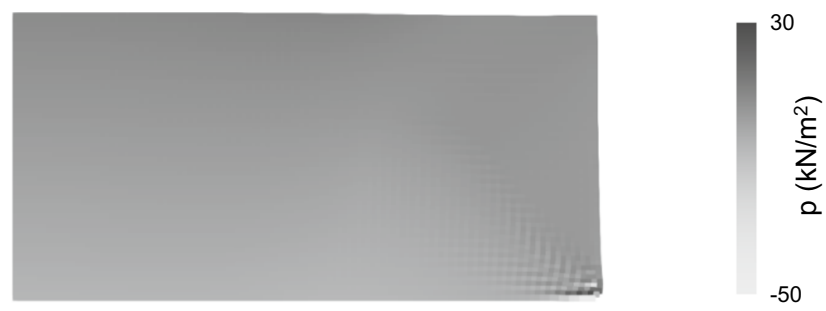

(f)
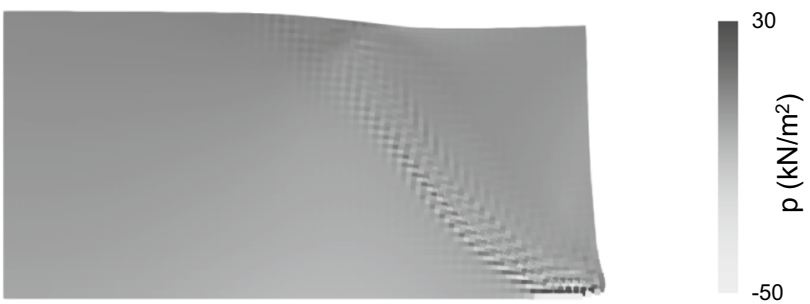

(g)

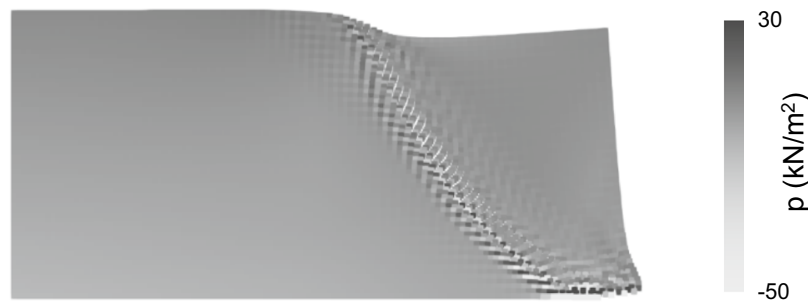

(h)
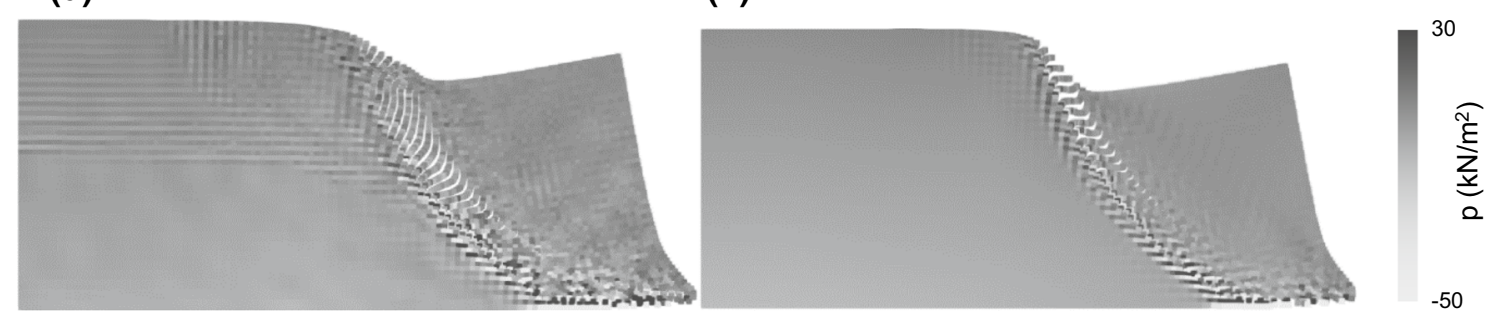

Fig. 29 Mean stress in the body using regular MPM (a-d) and DM-GC (e-h) after a horizontal toe displacement of a \& e $0.10 \mathrm{~m}, \mathbf{b} \& \mathbf{f} 0.30 \mathrm{~m}$, c \& $\mathbf{g} 0.50 \mathrm{~m}$, and $\mathbf{d} \& \mathbf{h} 1.0 \mathrm{~m}$

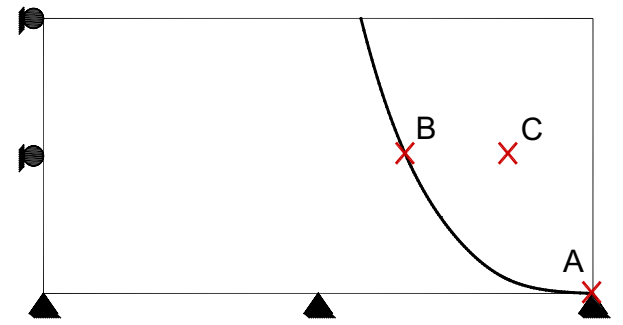

Fig. 30 Material points selected to plot stresses in p-q space

C, if regular MPM is used (Fig. 31b, c), the oscillations are extreme. It is evident that were a constitutive model different from Von Mises to be used, in which plasticity does not depend only on the deviatoric stress, regular MPM would not perform well. On the other hand, using DM-GC, the stress path appears to be well-behaved (Fig. 31e, f), with only some small oscillations.

Based on the results obtained with the benchmarks, Table 2 summarises the advantages and disadvantages of each of the methods studied in this paper.

\section{Conclusion}

MPM is a technique that is able to handle problems involving large deformations, since material properties and the body geometry are no longer attached to a mesh. 
Fig. 31 p-q curves using MPM $(\mathbf{a}-\mathbf{c})$, and DM-GC $(\mathbf{d}-\mathbf{f})$
DM-GC (a) Material point $\mathrm{A}$

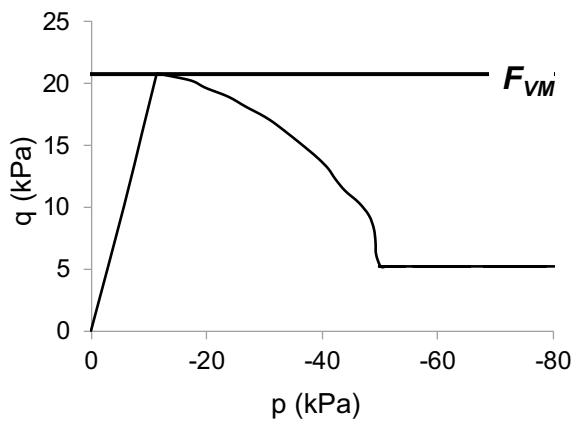

(b) Material point $B$

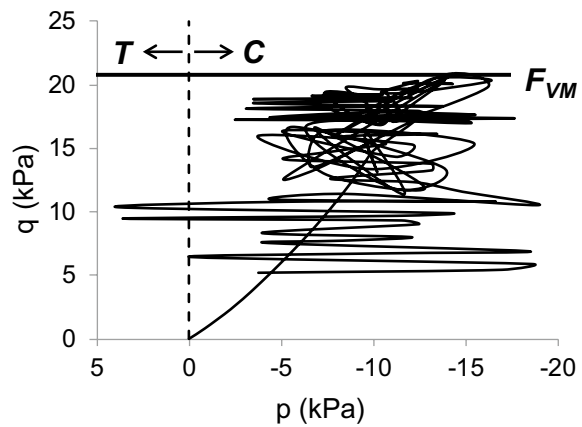

(c) Material point $\mathrm{C}$

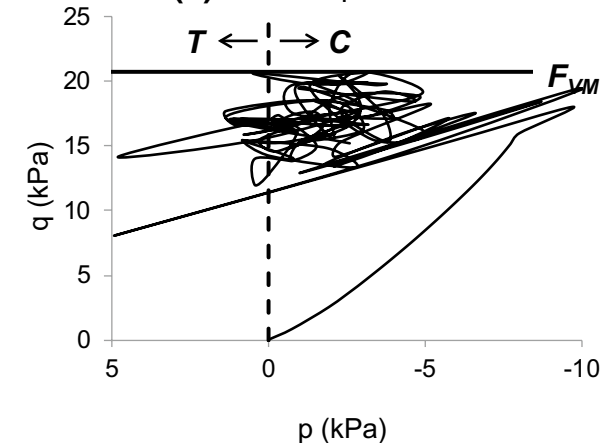

(d) Material point $\mathrm{A}$

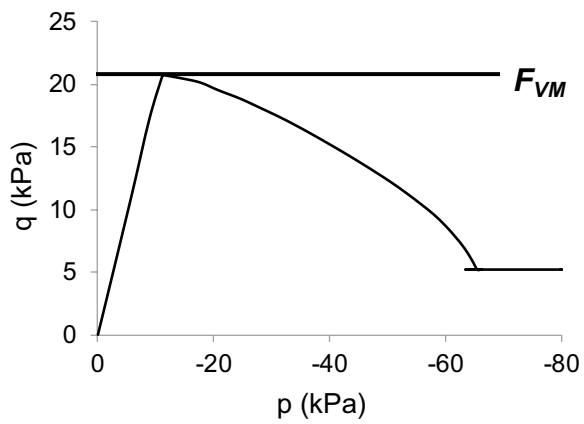

(e) Material point $B$

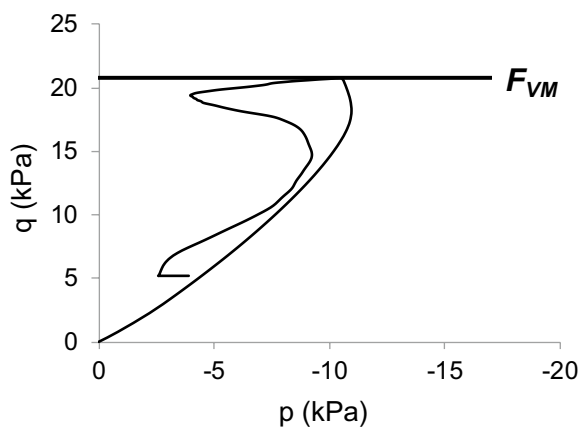

(f) Material point $\mathrm{C}$

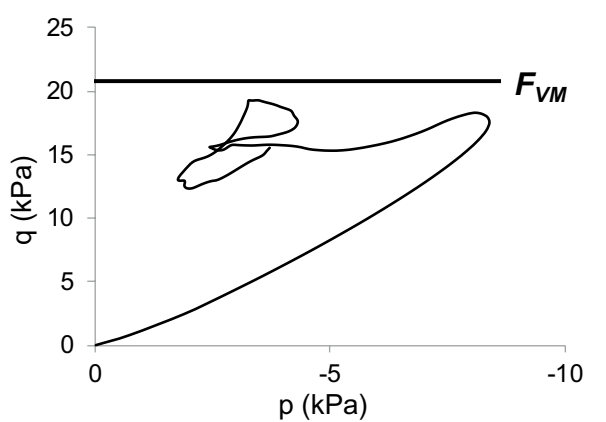

Unfortunately, the use of regular bi-linear finite element shape functions causes significant oscillations when integrating internal forces and stiffness, decreasing the accuracy of the simulations. Moreover, the grid crossing of a material point between elements and poor stress recovery create additional oscillations. A series of improvements, both novel and building upon the work of others, have been studied and combined to obtain an almost oscillation free version of MPM. It has been shown that GIMP reduces the errors caused by grid crossing, but integration using SF gradients, shown via an example using the stiffness matrix, is inaccurate due to the use of SF gradients that drop to zero at the inter-element boundaries. Using
GIMP together with a double mapping integration procedure significantly reduces the stiffness matrix oscillation. Also, it has been proven that CMPM increases the accuracy of the stresses computed at the material points compared to typical MPM and GIMP. These techniques combined (termed DM-GC) increases considerably the accuracy of the MPM simulations. Moreover, since it has been observed that DM performs well when using typical finite element shape functions, and better still when using GIMP shape functions, the combination of DM with other C1-continuous methods, such as CPDI, B-spline MPM or DDMP, is a possibility which can be studied in the future. The DM and DM-G methods have the benefit of being 
Table 2 Summary of advantages and disadvantages of the methods studied

\begin{tabular}{|c|c|c|c|c|c|}
\hline \multirow[t]{2}{*}{ Method } & \multicolumn{2}{|c|}{ Oscillations of nodal $\mathbf{F}^{\text {int }}$ caused by } & \multicolumn{2}{|l|}{ Oscillations of $\mathbf{K}$ caused by } & \multirow{2}{*}{$\begin{array}{l}\text { Stress recovery } \\
-\end{array}$} \\
\hline & MP position & MP CEB & MP position & MP CEB & \\
\hline MPM & $\begin{array}{l}\text { Inaccurate distribution of } \\
\text { internal forces }\end{array}$ & $\begin{array}{l}\text { Large oscillations due } \\
\text { to discontinuity of SF } \\
\text { gradients }\end{array}$ & $\begin{array}{l}\text { Increase if MP is close to } \\
\text { the node, and decrease } \\
\text { in some directions if } \\
\text { MP is far from the } \\
\text { node }\end{array}$ & $\begin{array}{l}\text { Accumulation or reduc- } \\
\text { tion of MPs causes an } \\
\text { increase or reduction } \\
\text { of stiffness }\end{array}$ & Poor \\
\hline GIMP & $\begin{array}{l}\text { Decrease of oscillation } \\
\text { by keeping continuity } \\
\text { of SF gradients }\end{array}$ & $\begin{array}{l}\text { Imbalance disappears } \\
\text { because of continuity } \\
\text { of SF gradients }\end{array}$ & $\begin{array}{l}\text { Large stiffness oscilla- } \\
\text { tion }\end{array}$ & $\begin{array}{l}\text { Stiffness vanishes if MP } \\
\text { is at element boundary }\end{array}$ & $\begin{array}{l}\text { Continuous between } \\
\text { elements }\end{array}$ \\
\hline DM-MPM & $\begin{array}{l}\text { Inaccurate distribution of } \\
\text { internal forces }\end{array}$ & $\begin{array}{l}\text { Large imbalance due to } \\
\text { discontinuity of SF } \\
\text { gradients }\end{array}$ & $\begin{array}{l}\text { Stiffness oscillation } \\
\text { reduces }\end{array}$ & $\begin{array}{l}\text { Stiffness oscillation } \\
\text { reduces significantly } \\
\text { by using the modified } \\
\text { weighting value } \mathrm{W}^{*}\end{array}$ & Poor \\
\hline DM-G & $\begin{array}{l}\text { Decrease of oscillation } \\
\text { by keeping continuity } \\
\text { of SF gradients }\end{array}$ & $\begin{array}{l}\text { Imbalance disappears } \\
\text { because of continuity } \\
\text { of SF gradients }\end{array}$ & $\begin{array}{l}\text { Stiffness oscillation } \\
\text { reduces significantly }\end{array}$ & $\begin{array}{l}\text { Stiffness oscillation } \\
\text { reduces significantly }\end{array}$ & $\begin{array}{l}\text { Continuous between } \\
\text { elements }\end{array}$ \\
\hline DM-GC & $\begin{array}{l}\text { Decrease of oscillation } \\
\text { by keeping continuity } \\
\text { of SF gradients }\end{array}$ & $\begin{array}{l}\text { Imbalance disappears } \\
\text { because of continuity } \\
\text { of SF gradients }\end{array}$ & $\begin{array}{l}\text { Stiffness oscillation } \\
\text { reduces significantly }\end{array}$ & $\begin{array}{l}\text { Stiffness oscillation } \\
\text { reduces significantly }\end{array}$ & $\begin{array}{l}\text { Highly improved by } \\
\text { increasing the solu- } \\
\text { tion domain and SF } \\
\text { order }\end{array}$ \\
\hline
\end{tabular}

MP: material point, $\mathrm{CEB}$ : crossing element boundary

able to be implemented implicitly or explicitly with typical elasto-plastic constitutive models.

Acknowledgements The authors of this work express their gratitude to the Mexican National Council for Science and Technology (CONACYT) for financing this work through the Grant Number 409778.

Open Access This article is distributed under the terms of the Creative Commons Attribution 4.0 International License (http://creativeco mmons.org/licenses/by/4.0/), which permits unrestricted use, distribution, and reproduction in any medium, provided you give appropriate credit to the original author(s) and the source, provide a link to the Creative Commons license, and indicate if changes were made.

\section{Appendices}

\section{Appendix A1: Plane strain and axisymmetric matrices}

For plane strain conditions, the $\mathbf{B}$ and $\mathbf{D}$ matrices are

$$
\begin{aligned}
& \mathbf{B}=\left[\begin{array}{cccccccc}
\frac{\partial N_{1}}{\partial x} & 0 & \frac{\partial N_{2}}{\partial x} & 0 & \frac{\partial N_{3}}{\partial x} & 0 & \frac{\partial N_{4}}{\partial x} & 0 \\
0 & \frac{\partial N_{1}}{\partial y} & 0 & \frac{\partial N_{2}}{\partial y} & 0 & \frac{\partial N_{3}}{\partial y} & 0 & \frac{\partial N_{4}}{\partial y} \\
\frac{\partial N_{1}}{\partial y} & \frac{\partial N_{1}}{\partial x} & \frac{\partial N_{2}}{\partial y} & \frac{\partial N_{2}}{\partial x} & \frac{\partial N_{3}}{\partial y} & \frac{\partial N_{3}}{\partial x} & \frac{\partial N_{4}}{\partial y} & \frac{\partial N_{4}}{\partial x}
\end{array}\right] \\
& \mathbf{D}=\frac{E(1-\nu)}{(1+\nu)(1-2 \nu)}\left[\begin{array}{ccc}
1 & \frac{\nu}{1-\nu} & 0 \\
\frac{\nu}{1-\nu} & 1 & 0 \\
0 & 0 & \frac{1-2 \nu}{2(1-\nu)}
\end{array}\right]
\end{aligned}
$$

For axisymmetric conditions, cylindrical coordinates are used, and after integration over one radian the stiffness matrix becomes

$\mathbf{K}=\sum_{\mathrm{p}=1}^{\mathrm{nmp}} \mathbf{B}^{\mathrm{axT}}\left(\mathbf{x}_{\mathrm{p}}\right) \mathbf{D}_{\mathrm{p}}^{\mathrm{ax}} \mathbf{B}^{\mathrm{ax}}\left(\mathbf{x}_{\mathrm{p}}\right)|\mathbf{J}| \mathrm{W} \cdot \mathrm{r}_{\mathrm{p}}$

The element (nodal) internal forces $\mathbf{F}^{\text {int }}$ are

$\mathbf{F}^{\text {int }}=\sum_{\mathrm{p}=1}^{\mathrm{nmp}} \boldsymbol{\sigma}_{\mathrm{p}}^{\mathrm{ax}} \mathbf{B}^{\mathrm{ax}}\left(\mathbf{x}_{\mathrm{p}}\right)|\mathbf{J}| \mathrm{W} \cdot \mathrm{r}_{\mathrm{p}}$

and the element (nodal) external forces $\mathbf{F}^{\text {ext }}$ considering only gravity are

$\mathbf{F}^{\text {ext }}=\sum_{\mathrm{p}=1}^{\mathrm{nmp}} \rho_{\mathrm{p}} \mathbf{N}^{\mathrm{T}}\left(\mathbf{x}_{\mathrm{p}}\right) \mathbf{g}|\mathbf{J}| \mathrm{W} \cdot \mathrm{r}_{\mathrm{p}}$

where

$\mathbf{B}^{\mathrm{ax}}=\left[\begin{array}{cccccccc}\frac{\partial \mathrm{N}_{1}}{\partial \mathrm{r}} & 0 & \frac{\partial \mathrm{N}_{2}}{\partial \mathrm{r}} & 0 & \frac{\partial \mathrm{N}_{3}}{\partial \mathrm{r}} & 0 & \frac{\partial \mathrm{N}_{4}}{\partial \mathrm{r}} & 0 \\ 0 & \frac{\partial \mathrm{N}_{1}}{\partial \mathrm{y}} & 0 & \frac{\partial \mathrm{N}_{2}}{\partial \mathrm{y}} & 0 & \frac{\partial \mathrm{N}_{3}}{\partial \mathrm{y}} & 0 & \frac{\partial \mathrm{N}_{4}}{\partial \mathrm{y}} \\ \frac{\partial \mathrm{N}_{1}}{\partial \mathrm{y}} & \frac{\partial \mathrm{N}_{1}}{\partial \mathrm{r}} & \frac{\partial \mathrm{N}_{2}}{\partial \mathrm{y}} & \frac{\partial \mathrm{N}_{2}}{\partial \mathrm{r}} & \frac{\partial \mathrm{N}_{3}}{\partial \mathrm{y}} & \frac{\partial \mathrm{N}_{3}}{\partial \mathrm{r}} & \frac{\partial \mathrm{N}_{4}}{\partial \mathrm{y}} & \frac{\partial \mathrm{N}_{4}}{\partial \mathrm{r}} \\ \frac{\mathrm{N}_{1}}{\mathrm{r}} & 0 & \frac{\mathrm{N}_{2}}{\mathrm{r}} & 0 & \frac{\mathrm{N}_{3}}{\mathrm{r}} & 0 & \frac{\mathrm{N}_{4}}{\mathrm{r}} & 0\end{array}\right]$ 
$\mathbf{D}^{\mathrm{ax}}=\frac{\mathrm{E}(1-\nu)}{(1+\nu)(1-2 \nu)}\left[\begin{array}{cccc}1 & \frac{\nu}{1-\nu} & 0 & \frac{\nu}{1-\nu} \\ \frac{\nu}{1-\nu} & 1 & 0 & \frac{\nu}{1-\nu} \\ 0 & 0 & \frac{1-2 \nu}{2(1-\nu)} & 0 \\ \frac{\nu}{1-\nu} & \frac{\nu}{1-\nu} & 0 & 1\end{array}\right]$

where $\mathbf{B}^{\text {ax }}$ refers to the axisymmetric strain-displacement matrix, $\mathbf{D}^{\text {ax }}$ refers to the stress-strain matrix, and $r_{p}$ is the radial distance between the sampling point and the axisymmetric axis.

\section{Appendix A2: Analytical axisymmetric solution}

The radial, tangential and axial stress distributions $\left(\sigma_{\mathrm{r}}, \sigma_{\theta}\right.$ and $\sigma_{y}$, respectively) in the wall of a hollow cylinder at a radius $r$ from the cylinder axis are computed as

$\sigma_{\mathrm{r}}=\frac{\mathrm{A}}{\mathrm{r}^{2}}+2 \mathrm{C}$

$\sigma_{\theta}=-\frac{\mathrm{A}}{\mathrm{r}^{2}}+2 \mathrm{C}$

$\sigma_{\mathrm{y}}=4 \nu \mathrm{C}$

where $\mathrm{A}$ and $\mathrm{C}$ are constants given by

$\mathrm{A}=2(1-2 v) \mathrm{Cr}_{\mathrm{e}}^{2}$

$\mathrm{C}=\frac{\mathrm{EA}_{1}}{2(1+\nu)(1-2 \nu)}$

in which $\mathrm{A}_{1}$ is a function of the boundary conditions. For a cylinder that is fixed at the external boundary $\left(r_{e}\right)$ and loaded at the internal boundary by a pressure $\mathrm{p}_{\mathrm{s}}$,

$A_{1}=\frac{p_{s}(1+\nu)(1-2 \nu)}{r_{e}^{2} E\left(-\frac{(1-2 \nu)}{r_{i}^{2}}-\frac{1}{r_{e}^{2}}\right)}$

For more details regarding this analytical axisymmetric solution, the reader is directed to Popov [22].

\section{Appendix B: Conservation of mass and momentum of implicit MPM}

In MPM, because of the partition of unity of the SFs, mass is automatically conserved in mapping:

$\sum_{\mathrm{i}}^{\mathrm{nn}} \mathrm{m}_{\mathrm{i}}=\sum_{\mathrm{i}}^{\mathrm{nn}} \sum_{\mathrm{p}=1}^{\mathrm{nmp}} \mathrm{m}_{\mathrm{p}} \mathrm{N}_{\mathrm{i}}\left(\mathbf{x}_{\mathrm{p}}\right)=\sum_{\mathrm{p}=1}^{\mathrm{nmp}} \mathrm{m}_{\mathrm{p}}$

where i denotes the node.

Considering the conservation of momentum, the method uses the FEM approach to solve the equation of motion on the nodes, which conserves momentum, and therefore it is the updating of the material point momentum that is considered here. The total momentum of a material point at the end of a time step is used considering Newmark's scheme, which is computed as

$\sum_{p=1}^{n m p} m_{p} \mathbf{v}_{p}^{t+\Delta t}=\sum_{p=1}^{n m p} m_{p}\left[\mathbf{v}_{p}^{t}+\frac{1}{2}\left[\mathbf{a}_{p}^{t}+\sum_{i=1}^{n n} \overline{\mathbf{a}}^{t+\Delta t} N_{i}\left(\mathbf{x}_{p}\right)\right]\right]$

where $\mathbf{v}_{\mathrm{p}}$ and $\mathbf{a}_{\mathrm{p}}$ are the velocity and accelerations, respectively, at the material points and

$\overline{\mathbf{a}}_{\mathrm{i}}^{\mathrm{t}+\Delta \mathrm{t}}=\frac{4 \Delta \overline{\mathbf{u}}_{\mathrm{i}}}{\Delta \mathrm{t}^{2}}-\frac{4 \overline{\mathbf{v}}_{\mathrm{i}}^{\mathrm{t}}}{\Delta \mathrm{t}}-\overline{\mathbf{a}}_{\mathrm{i}}^{\mathrm{t}}$

In MPM, the total nodal momentum and change of momentum are equal to the total material point momentum and change of momentum, due to the partition of unity of the SF, as

$\sum_{i=1}^{n n} m_{i} \overline{\mathbf{v}}_{i}^{t}=\sum_{i=1}^{n n} \sum_{p=1}^{n m p} m_{p} \mathbf{v}_{p}^{t} N_{i}\left(\mathbf{x}_{p}\right)=\sum_{p=1}^{n m p} m_{p} \mathbf{v}_{p}^{t}$

$\sum_{i=1}^{n n} m_{i} \mathbf{a}_{i}^{t}=\sum_{i=1}^{n n} \sum_{p=1}^{n m p} m_{p} \mathbf{a}_{p}^{t} N_{i}\left(\mathbf{x}_{p}\right)=\sum_{p=1}^{n m p} m_{p} \mathbf{a}_{p}^{t}$

Substituting Eqs. 44 and 43 into Eq. 41 and rearranging loads to

$$
\begin{aligned}
\sum_{p=1}^{n m p} m_{p} \mathbf{v}_{p}^{t+\Delta t} & =\sum_{p=1}^{n m p} m_{p} \mathbf{v}_{p}^{t}+\frac{1}{2} \Delta t \sum_{p=1}^{n m p} m_{p} \mathbf{a}_{p}^{t}+2 \sum_{i=1}^{n n} m_{i} \frac{\Delta \overline{\mathbf{u}}_{i}}{\Delta t} \\
& -2 \sum_{i=1}^{n n} m_{i} \overline{\mathbf{v}}_{i}^{t}-\frac{1}{2} \Delta t \sum_{p=1}^{n m p} m_{p} \mathbf{a}_{p}^{t}
\end{aligned}
$$

which reduces further to

$\sum_{p=1}^{n m p} m_{p} \mathbf{v}_{p}^{t+\Delta t}=2 \sum_{i=1}^{n n} m_{i} \frac{\Delta \overline{\mathbf{u}}_{i}}{\Delta t}-\sum_{i=1}^{n n} m_{i} \overline{\mathbf{v}}_{i}^{t}$

where $\Delta \overline{\mathbf{u}}_{\mathrm{i}}$ is computed using Eq. 10, which is extended using the Newton-Raphson iteration procedure as

$\left(\mathbf{K}^{\mathrm{t}}+\frac{4 \mathbf{M}^{\mathrm{t}}}{\Delta \mathrm{t}^{2}}\right) \delta \overline{\mathbf{u}}=\mathbf{F}_{\mathrm{ext}}^{\mathrm{t}+\Delta \mathrm{t}}+\mathbf{M}^{\mathrm{t}}\left(-\frac{4 \Delta \overline{\mathbf{u}}^{\mathrm{k}-1}}{\Delta \mathrm{t}^{2}}+\frac{4}{\Delta \mathrm{t}} \overline{\mathbf{v}}^{\mathrm{t}}+\overline{\mathbf{a}}^{\mathrm{t}}\right)-\mathbf{F}_{\mathrm{int}}^{\mathrm{t}+\Delta \mathrm{t}}$

and

$\Delta \overline{\mathbf{u}}^{\mathrm{k}}=\Delta \overline{\mathbf{u}}^{\mathrm{k}-1}+\delta \overline{\mathbf{u}}$

where $\Delta \overline{\mathbf{u}}^{\mathrm{k}}$ is the displacement within an iteration step, $\delta \overline{\mathbf{u}}$ is the incremental displacement, and $\mathrm{k}$ is the iteration number. After Eq. 47 reaches convergence (i.e. the right hand side 
reduces to zero within a specified tolerance), the following is true:

$\mathbf{F}_{\text {ext }}^{\mathrm{t}+\Delta \mathrm{t}}+\mathbf{M}^{\mathrm{t}}\left(-\frac{4 \Delta \overline{\mathbf{u}}}{\Delta \mathrm{t}^{2}}+\frac{4}{\Delta \mathrm{t}} \overline{\mathbf{v}}^{\mathrm{t}}+\overline{\mathbf{a}}^{\mathrm{t}}\right)-\mathbf{F}_{\text {int }}^{\mathrm{t}+\Delta \mathrm{t}}=\{0\}$

Considering an isolated system, i.e. where momentum would not be altered by external forces, it can be stated that

$\mathbf{F}_{\mathrm{ext}}^{\mathrm{t}+\Delta \mathrm{t}}=\{0\}$

At the beginning of the time step for an isolated system, there is no net rate of change of momentum:

$\sum_{\mathrm{p}=1}^{\mathrm{nmp}} \mathrm{m}_{\mathrm{p}} \mathbf{a}_{\mathrm{p}}^{\mathrm{t}}=\sum_{\mathrm{i}=1}^{\mathrm{nn}} \mathrm{m}_{\mathrm{i}} \overline{\mathbf{a}}_{\mathrm{i}}^{\mathrm{t}}=\sum_{\mathrm{i}}^{\mathrm{nn}} \sum_{\mathrm{j}}^{\mathrm{nn}} \mathrm{m}_{\mathrm{ij}} \overline{\mathbf{a}}_{\mathrm{j}}^{\mathrm{t}}=\{0\}$

where $\mathrm{m}_{\mathrm{ij}}$ is equivalent to $\mathbf{M}^{\mathrm{t}}$. Moreover, acknowledging that $\sum_{\mathrm{i}}^{\mathrm{nn}} \mathbf{B}_{\mathrm{i}}\left(\mathbf{x}_{\mathrm{p}}\right)=\{0\}$, then

$\sum_{\mathrm{i}=1}^{\mathrm{nn}} \mathbf{F}_{\mathrm{i}, \text { int }}^{\mathrm{t}+\Delta \mathrm{t}}=-\sum_{\mathrm{i}=1}^{\mathrm{nn}} \sum_{\mathrm{p}=1}^{\mathrm{nmp}} \boldsymbol{\sigma}_{\mathrm{p}} \mathbf{B}_{\mathrm{i}}\left(\mathbf{x}_{\mathrm{p}}\right)|\mathbf{J}| \mathrm{W}=\{0\}$

Summing Eq. 49 over all nodes, and substituting in Eq. 50-52, yields $\sum_{i=1}^{n n} \sum_{j=1}^{n n} m_{i j} \frac{4 \Delta \overline{\mathbf{u}}_{j}}{\Delta t^{2}}-\sum_{i=1}^{n n} \sum_{j=1}^{n n} m_{i j} \frac{4 \Delta \overline{\mathbf{v}}_{j}}{\Delta t}=\{0\}$

Finally, substituting Eq. 53 into Eq. 46 leads to the conservation of momentum for the isolated system as

$\sum_{p=1}^{n m p} m_{p} \mathbf{v}_{p}^{t+\Delta t}=2 \sum_{i=1}^{n n} m_{i} \bar{v}_{i}^{t}-\sum_{i=1}^{n n} m_{i} \bar{v}_{i}^{t}=\sum_{p=1}^{n m p} m_{p} \mathbf{v}_{p}^{t}$

Note that the previous elaboration also holds for DM, DM-G and DM-GC, because (1) the modified stiffness matrix $\left(\mathbf{K}^{\dagger}\right)$ does not affect the conservation of momentum, and (2) the GIMP and CMPM SFs satisfy the partition of unity and Eq. 52.

\section{Appendix C: Double mapping procedures}

Figure 32 summarises the steps to perform stiffness integration using DM techniques (DM-MPM and DM-G). It is highlighted that both the DM and DM-G techniques follow the same steps. Making the following minor modifications, it is possible change between DM and DM-G: (i) Loop 3 loops over the material points affecting the element, which can be either all material points in the element $(\mathrm{cmp})$ or all material
Fig. 32 Summary of the steps followed to perform DM stiffness integration

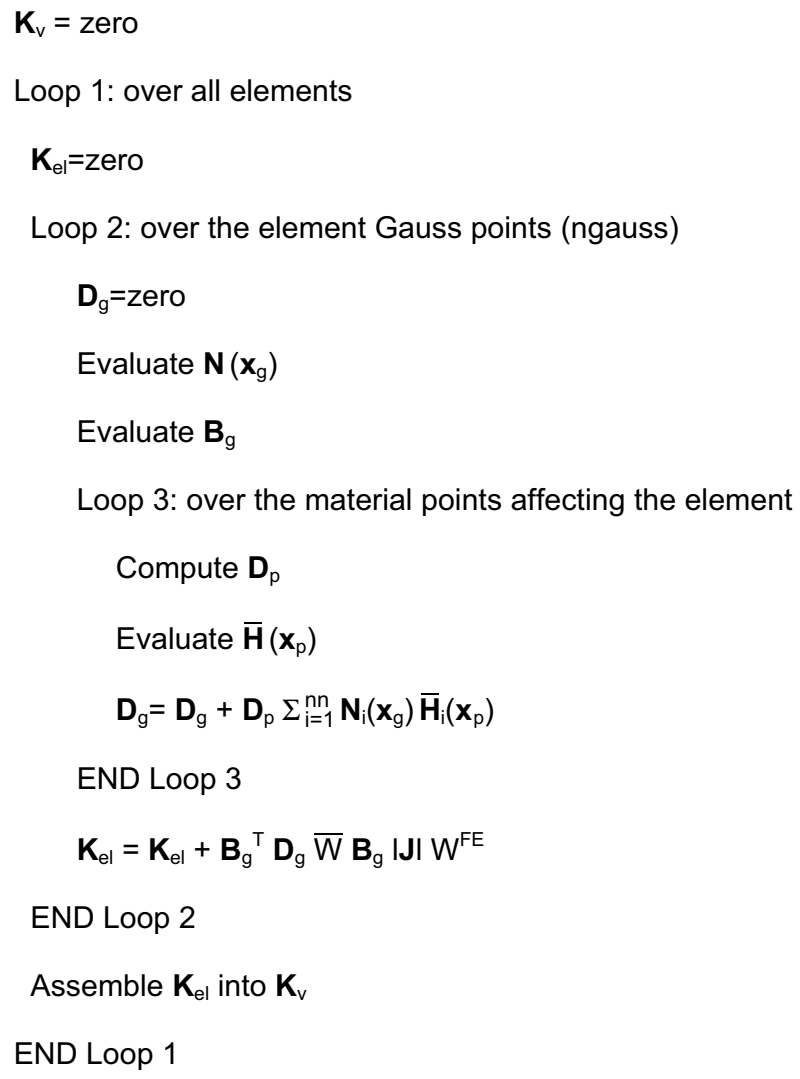


Fig. 33 Stress recovery steps using CMPM

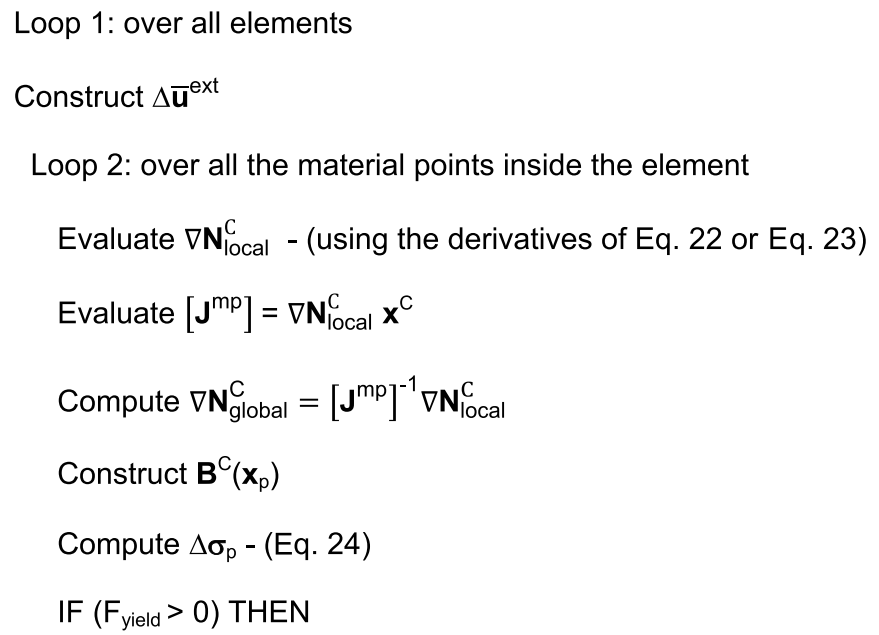

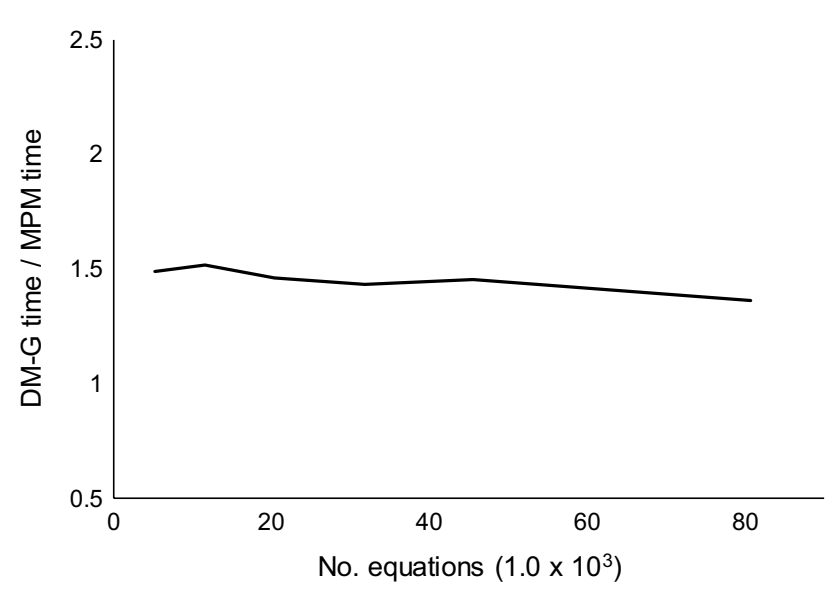

Fig. 34 Relationship between the computational times using DM-G and typical MPM

points with (part of) their support domain in the element (smp), for DM and DM-G respectively, (ii) the nodal shape function $\overline{\mathbf{H}}_{\mathrm{i}}$ represents $\mathrm{N}_{\mathrm{i}}$ or $\mathrm{S}_{\mathrm{ip} *}$, for DM and DM-G respectively, and (iii) the material point weight $\overline{\mathrm{W}}$ represents $\mathrm{W}^{*}$ or W, for DM and DM-G respectively. Summarizing, DM uses cmp, $\mathrm{N}_{\mathrm{i}}$ and $\mathrm{W}^{*}$, whereas DM-G uses smp, $\mathrm{S}_{\mathrm{ip}}$ and $\mathrm{W}$.

In Fig. 33, the stress recovery steps are shown considering CMPM. It is highlighted that all matrices are related to the patch used, so are larger than in regular MPM. For example, for 4 noded elements the patch is $4 \times 4$ nodes, instead of $2 \times 2$.
To demonstrate the computational performance of the DM-G algorithm (which has a higher computational cost when compared to DM), a series of tests were conducted on square meshes, with the results presented in Fig. 34. The tests consisted of computing the stiffnesses of meshes made up of $50,75,100,150$, and 200 elements per side, with each element filled with 4 equally distributed MPs. Each stiffness cycle was computed 200 times to obtain an accurate mean value of the time taken. In Fig. 34, the relationship between computational time using DM-G and regular MPM is plotted as a function of the number of equations. It is observed that the relationship is almost constant, with DM-G taking about 50\% longer than regular MPM. However, the overall increase of computational time for the problems studied is close to $5 \%$, although this is dependent on the solver and characteristics of the problem solved.

\section{References}

1. Alonso EE, Yerro A, Pinyol NM (2015) Recent developments of the material point method for the simulation of landslides. IOP Conf Ser Earth Environ Sci 26:1-32

2. Al-Kafaji IKJ (2013) Formulation of a dynamic material point method (MPM) for geomechanical problems. Ph.D. thesis, Stuttgart University

3. Andersen S, Andersen L (2009) Analysis of stress updates in the material-point method. Nordic Semin Comput Mech 11:129-134 
4. Bandara S, Soga K (2015) Coupling of soil deformation and pore fluid flow using material point method. Comput Geotech 63:199-214

5. Bardenhagen SG, Brackbill JU, Sulsky D (2000) The materialpoint method for granular materials. Comput Methods Appl Mech Engrg 187(3-4):529-541

6. Bardenhagen SG, Kober EM (2004) The generalized interpolation material point method. Comp Model Eng Sci 5(6):477-495

7. Barlow J (1976) Optimal stress locations in finite element models. Int J Numer Meth Engng 10(2):243-251

8. Bathe KJ (1996) Finite element procedures. Prentice Hall, New Jersey

9. Beuth L, Benz T, Vermeer PA, Więckowski Z (2008) Large deformation analysis using a quasi-static material point method. J Theor Appl Mech 38(1-2):45-60

10. Charlton TJ, Coombs WM, Augarde CE (2017) iGIMP: an implicit generalised interpolation material point method for large deformations. Comput Struct 190:108-125

11. Chen ZP, Zhang X, Qiu XM, Liu Y (2017) A frictional contact algorithm for implicit material point method. Comput Methods Appl Mech Engrg 321:124-144

12. Coetzee CJ, Vermeer PA, Basson AH (2005) The modelling of anchors using the material point method. Int J Numer Anal Meth Geomech 29(9):879-895

13. Coombs WM, Charlton TJ, Cortis M, Augarde CE (2018) Overcoming volumetric locking in material point methods. Comput Methods Appl Mech Engrg 333:1-21

14. González Acosta L, Vardon PJ, Hicks MA (2017) Composite material point method (CMPM) to improve stress recovery for quasi-static problems. Procedia Eng 175:324-331

15. González Acosta L, Vardon PJ, Hicks MA (2018) The use of MPM to estimate the behaviour of rigid structures during landslides. In: Proceedings of the 9th European conference on numerical methods in geotechnical engineering. Taylor \& Francis, Porto

16. Liang Y, Zhang X, Liu Y (2019) An efficient staggered grid material point method. Comput Methods Appl Mech Engrg 352:85-109

17. Lorenzo R, da Cunha RP, Cordão Neto MP, Nairn JA (2017) Numerical simulation of installation of jacked piles in sand using material point method. Can Geotech J 55(1):131-146

18. Mar A, Hicks MA (1996) A benchmark computational study of finite element error estimation. Int J Numer Meth Engng 39(23):3969-3983

19. Naylor DJ (1974) Stresses in nearly incompressible materials by finite elements with application to the calculation of excess pore pressure. Int J Numer Meth Engng 8(3):443-460

20. Newmark NM (1959) A method of computation for structural dynamics. J Eng Mech Div-ASCE 85(3):37-94
21. Phuong NTV, van Tol AF, Elkadi ASK, Rohe A (2016) Numerical investigation of pile effects in sand using material point method. Comput Geotech 73:58-71

22. Popov EP, Balan TA (1999) Engineering mechanics of solids, 2nd edn. Prentice Hall, New Jersey

23. Sadeghirad A, Astaneh AV (2011) A finite element method with composite shape functions. Eng Comput Int J Comput Aided Eng Softw 28(4):389-422

24. Sadeghirad A, Brannon RM, Burghardt J (2011) A convected particle domain interpolation technique to extend applicability of the material point method for problems involving massive deformations. Int J Numer Meth Engng 86(12):1435-1456

25. Solowski WT, Sloan SW (2015) Evaluation of material point method for use in geotechnics. Int J Numer Anal Meth Geomech 39(7):695-701

26. Steffen M, Kirby RM, Berzins M (2008) Analysis and reduction of quadrature errors in the material point method (MPM). Int J Numer Meth Engng 76(6):922-948

27. Steffen M, Wallstedt PC, Guilkey JE, Kirby RM, Berzins M (2008) Examination and analysis of implementation choices within the material point method (MPM). Comput Model Eng Sci 31(2):107-127

28. Sulsky D, Chen Z, Schreyer HL (1994) A particle method for history dependent materials. Comput Methods Appl Mech Engrg 118(1-2):179-196

29. Sulsky D, Kaul A (2004) Implicit dynamics in the material-point method. Comput Methods Appl Mech Engrg 193(12-14):1137-1170

30. Sulsky D, Zhou S, Schreyer HL (1995) Application of a particle-in-cell method to solid mechanics. Comput Phys Commun 87(1-2):236-252

31. Wang B, Vardon PJ, Hicks MA (2016) Investigation of retrogressive and progressive slope failure mechanisms using the material point method. Comput Geotech 78:88-98

32. Wang B, Vardon PJ, Hicks MA (2016) Development of an implicit material point method for geotechnical applications. Comput Geotech 71:159-167

33. Więckowski $Z$ (2004) The material point method in large strain engineering problems. Comput Methods Appl Mech Engrg 193(39-41):4417-4438

34. Zhang DZ, Ma X, Giguere PT (2011) Material point method enhanced by modified gradient of shape function. J Comput Phys 230(16):6379-6398

35. Zienkiewicz OC, Taylor RL, Zhu JZ (2013) The finite element method: Its basis and fundamentals, 7 th edn. Elsevier, Oxford

Publisher's Note Springer Nature remains neutral with regard to jurisdictional claims in published maps and institutional affiliations. 